
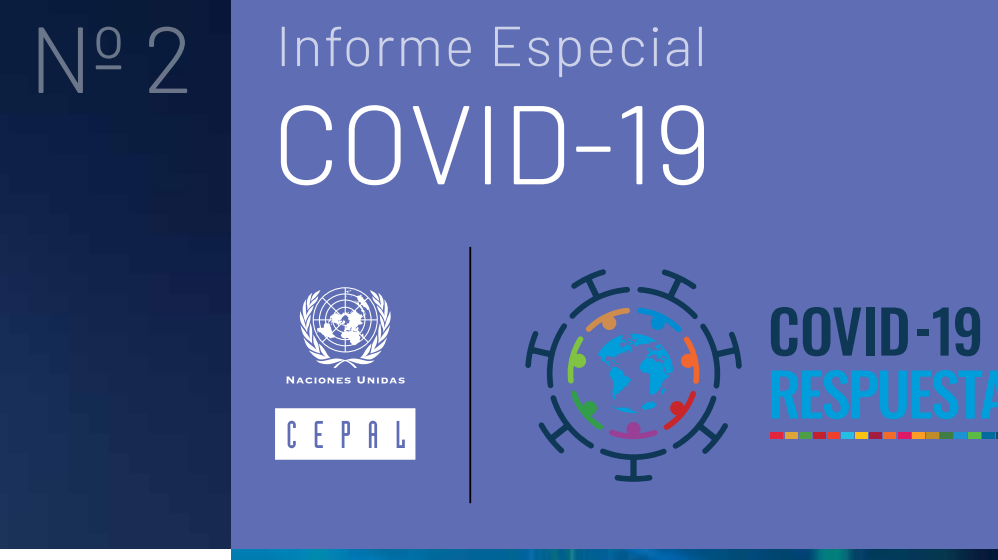

\title{
Dimensionar los efectos del COVID-19 para pensar en la reactivación
}

\section{A. Antes de la pandemia, América Latina y el Caribe tenía el menor crecimiento económico en décadas y un limitado espacio de política ${ }^{1}$}

- La pandemia del COVID-19 impactó América Latina y el Caribe en un momento de debilidad de su economía y de vulnerabilidad macroeconómica. En el decenio posterior a la crisis financiera mundial (2010-2019), la tasa de crecimiento del PIB regional disminuyó del $6 \%$ al $0,2 \%$; más aún, el período 2014-2019 fue el de menor crecimiento desde la década de $1950(0,4 \%)$ (véase el gráfico 1 y el primer Informe de esta serie).

- A medida que la pandemia se propaga en la región, su caracterización como crisis sanitaria, económica y social es cada vez más evidente. Por su parte, la dimensión y la duración de sus efectos, si bien difíciles de cuantificar debido a la incertidumbre, comienzan a ser percibidas con claridad. Será la causa de la mayor crisis económica y social de la región en décadas, con efectos muy negativos en el empleo, el combate a la pobreza y la reducción de la desigualdad.

- El objetivo de este segundo Informe Especial es dimensionar los efectos económicos de la pandemia en el corto y mediano plazo. Para el corto plazo, es decir en el curso de 2020, se presentan estimaciones de la dinámica de la producción, el empleo, la pobreza y la distribución del ingreso con base en la información disponible al 17 de abril de 2020, que abarca todas las subregiones de América Latina y el Caribe y muchas de sus economías. Asimismo, se detallan las principales medidas de política macroeconómicas para enfrentar los efectos de la pandemia anunciadas hasta esa fecha.

- Dimensionar la caída de la actividad económica permite comenzar a determinar la magnitud del esfuerzo para un regreso a la normalidad. Pero ese regreso no será y no debe ser una vuelta a la situación existente antes de la pandemia. La visión de mediano plazo con la que concluye este Informe ilustra los cambios estructurales en la organización de la actividad productiva que están en proceso y que se intensificarán. Esto además tendrá impactos, en ocasiones irreversibles, en la estructura laboral, el empleo y el bienestar.

- De la conjunción de las estimaciones cuantitativas de corto plazo y las previsiones sobre los principales cambios cualitativos de mediano plazo surgirá lo que será una nueva normalidad.

\section{Índice}

A. Antes de la pandemia, América Latina y el Caribe tenía el menor crecimiento económico en décadas y un limitado espacio de política

B. La pandemia llevará a la mayor contracción de la actividad económica en la historia de la región...

C. Respuestas de política para enfrentar los efectos económicos y sociales de la pandemia

D. Proyecciones de crecimiento de los países de América Latina y el Caribe en 2020 .... 15

E. Desafíos de la política macroeconómica para seguir avanzando en la mitigación de los efectos de la crisis.

F. Efectos estructurales de la pandemia: el mundo posterior al COVID-19 18

Bibliografía. 21 
Gráfico 1 | América Latina y el Caribe: evolución de la tasa de crecimiento del PIB real, 1951-2019 (En porcentajes)

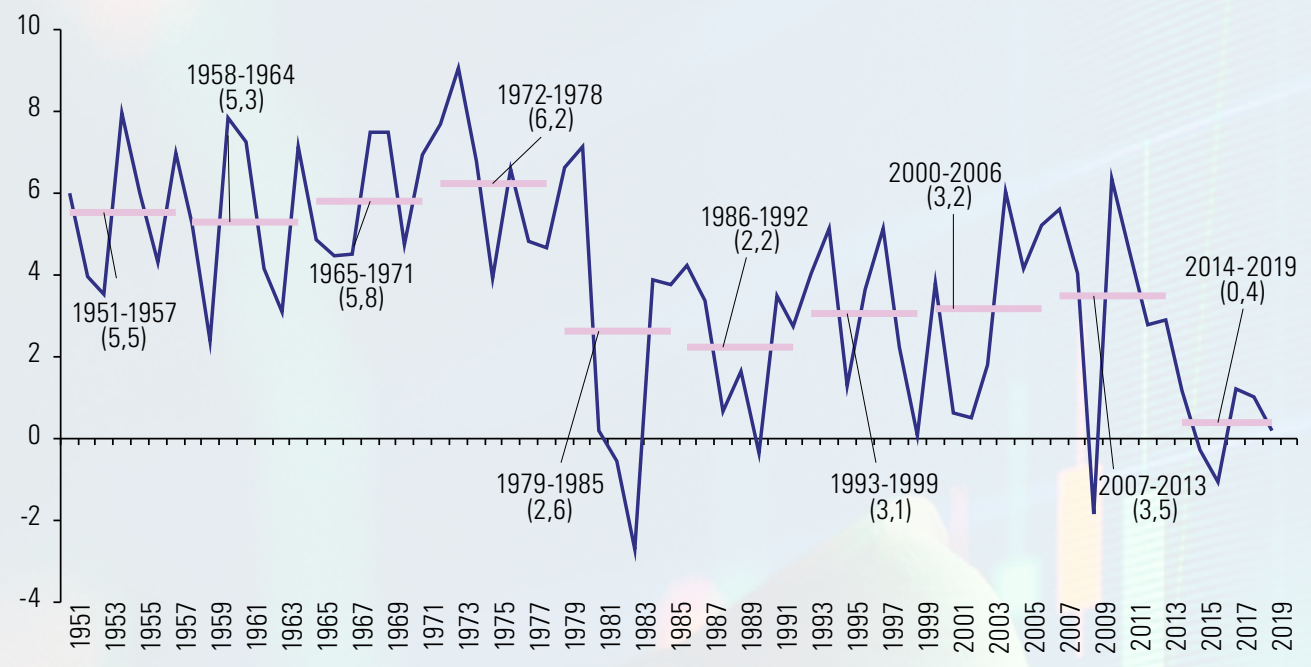

Fuente: Comisión Económica para América Latina y el Caribe (CEPAL), sobre la base de cifras oficiales.

\section{Una región con poco espacio para aumentar su gasto fiscal por el mayor endeudamiento, el creciente pago de intereses y los limitados ingresos fiscales}

- La acumulación de déficits fiscales en América Latina (2,7\% en promedio en la última década) aumentó la deuda pública bruta de los gobiernos centrales, que en 2019 promedió un 44,8\% del PIB, un incremento de 15 puntos porcentuales respecto a su mínimo del 29,8\% del PIB en 2011 (véase el gráfico 2). Existe gran heterogeneidad entre países, mientras el Paraguay y el Perú tenían niveles de endeudamiento inferiores al 25\% del PIB a finales de 2019, otros países presentaban niveles mucho mayores, que alcanzaban el 89,4\% en la Argentina, el 75,8\% en el Brasil y el 61,3\% en Costa Rica. El peso de la deuda no solo es notorio en los gobiernos centrales, sino también en las empresas públicas no financieras (véase el recuadro 1).

Gráfico 2 | América Latina (18 países): niveles de deuda pública bruta de los gobiernos centrales, 2000-2019 (En porcentajes del PIB)

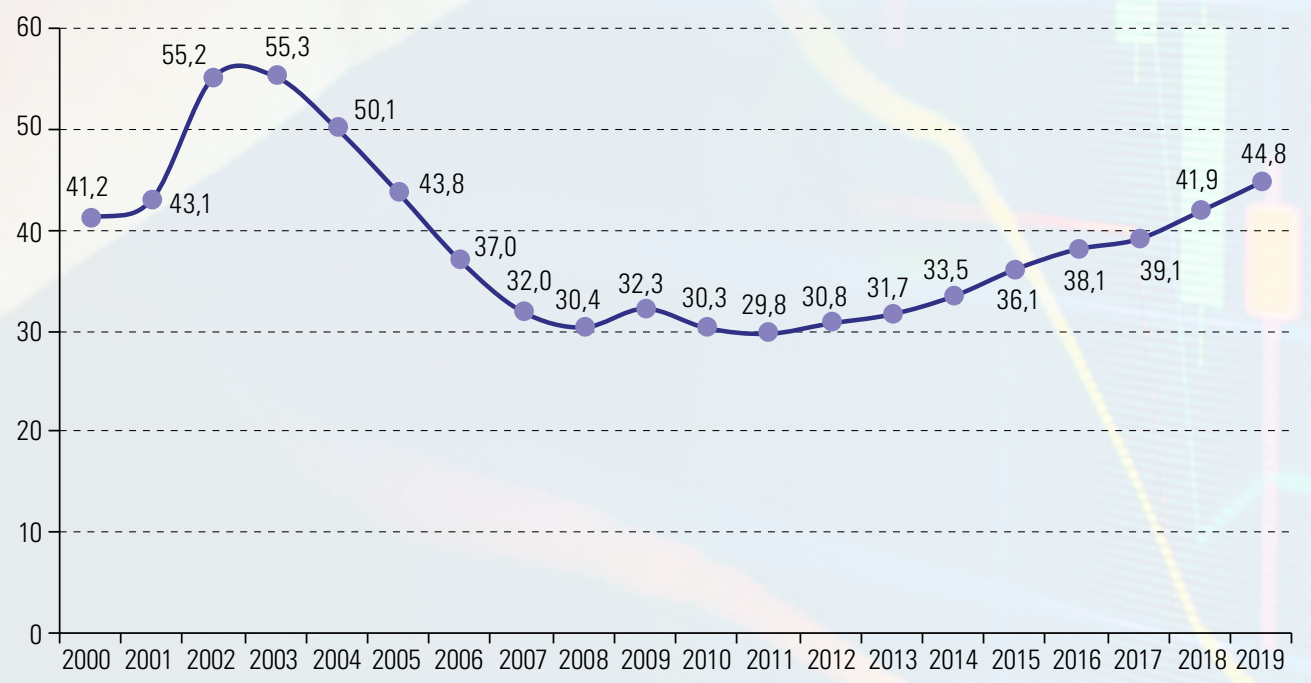

Fuente: Comisión Económica para América Latina y el Caribe (CEPAL), sobre la base de cifras oficiales. 
El aumento de la deuda en América Latina y el Caribe no solo afecta al gobierno central, sino también a las empresas del sector público no financiero, lo que aumenta la presión sobre el espacio de política fiscal. Estas empresas tienen una importancia estratégica por su tamaño, contribución a la recaudación fiscal y participación en la producción y las exportaciones. El aumento del endeudamiento afecta, en particular, a las empresas en los sectores de recursos naturales golpeadas por la disminución de los precios de las materias primas y las reducciones de ingresos y rentabilidad.

En promedio para la región en 2010-2016, el 42\% de las empresas del sector público no financiero presentaron pérdidas (netas de transferencias fiscales). Las transferencias requeridas para cubrir esas pérdidas estarian en un rango entre un tercio y un punto porcentual del PIB. La recapitalización de empresas del sector público no financiero requiere de esfuerzos financieros similares o mayores, con las consiguientes presiones adicionales al limitado espacio fiscal. Esta situación genera riesgos financieros importantes: Ios pasivos de las empresas del sector público no financiero representaron cerca del $9 \%$ del PIB, llegando en algunos casos a niveles de entre el $17 \%$ y el $20 \%$ del PIB (Pineda y Musacchio, 2020).

Fuente: Comisión Económica para América Latina y el Caribe (CEPAL).

- El aumento del pago de intereses redujo los recursos disponibles para el desarrollo como se evidencia en la evolución del gasto de los gobiernos en salud pública e inversiones. El pago de intereses aumentó del 1,7\% del PIB en 2010 al 2,6\% del PIB en 2019 (véase el gráfico 3), mientras que el gasto en salud tuvo un incremento mucho menor (del 1,9\% en 2010 al 2,3\% en 2018). Por su parte, el gasto de capital se redujo del $\mathbf{3 , 9} \%$ al $3,2 \%$ del PIB, y fue la variable de ajuste durante el proceso de consolidación fiscal observado entre 2016 y 2018. Una situación especialmente preocupante en materia de carga del servicio de la deuda se da en los países centroamericanos, como se muestra en el recuadro 2.

Gráfico 3 América Latina (16 países): pago de intereses, gastos de capital y gasto en salud de gobiernos centrales, 2010-2019a

(En porcentajes del PIB)

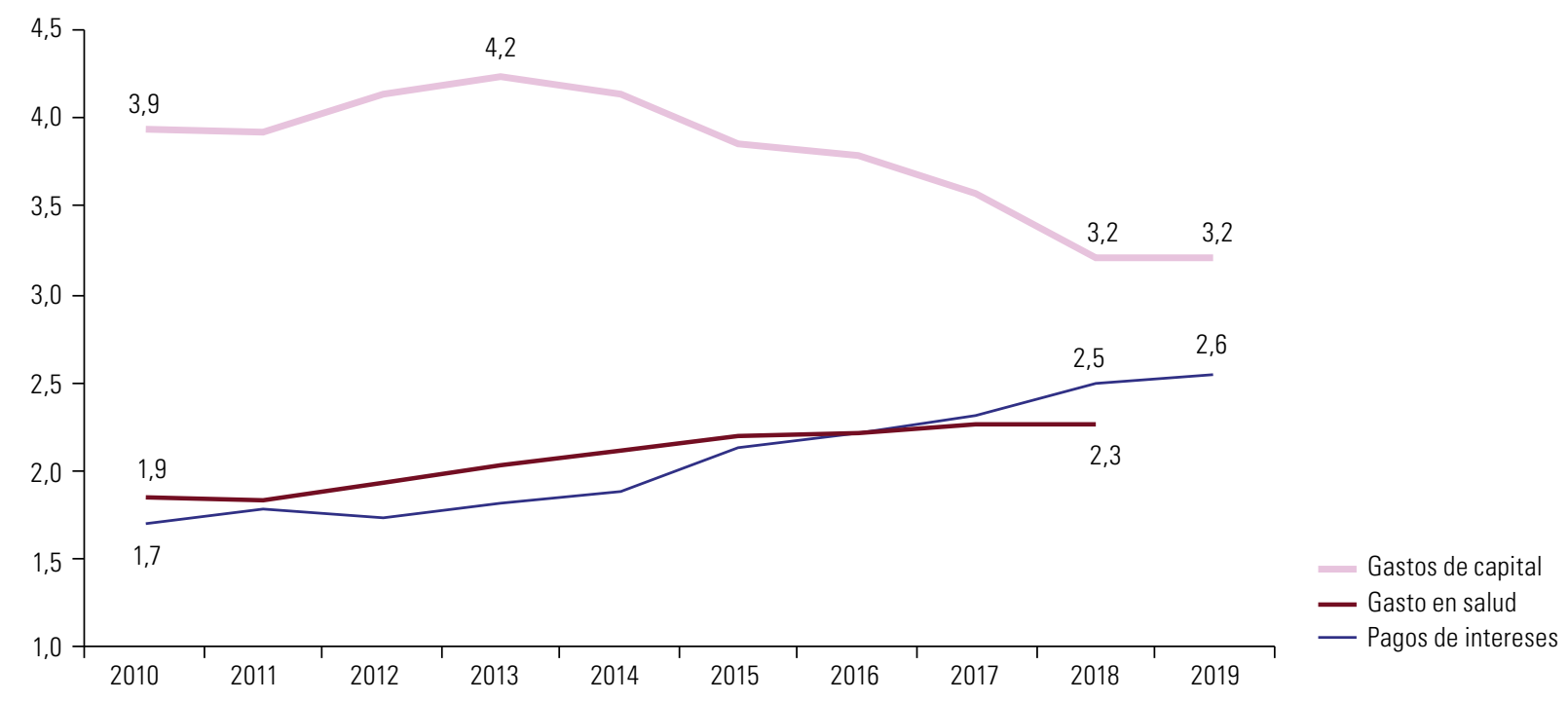

Fuente: Comisión Económica para América Latina y el Caribe (CEPAL), sobre la base de cifras oficiales.

a Los datos de gasto en salud son los disponibles hasta 2018. 


\section{Recuadro 2 | La carga del servicio de la deuda en Centroamérica traba la inversión pública y el gasto social}

En la última década, los paises de Centroamérica han buscado reducir la carga de su deuda mediante reformas fiscales (Costa Rica, Honduras) o la generación de superávits primarios (EI Salvador). Pese a ello, la deuda pública de los gobiernos centrales de los países incluidos en el gráfico aumentó de un promedio del 33,2\% del PIB en 2010 a uno del 44,0\% del PIB en 2019. Al mismo tiempo, los pagos de intereses crecieron del $\mathbf{1 , 8} \%$ del PIB al $\mathbf{2 , 5} \%$ del PIB.

Países seleccionados: deuda pública bruta y pagos de intereses de los gobiernos centrales, 2010 y 2019 (En porcentajes del PIB)

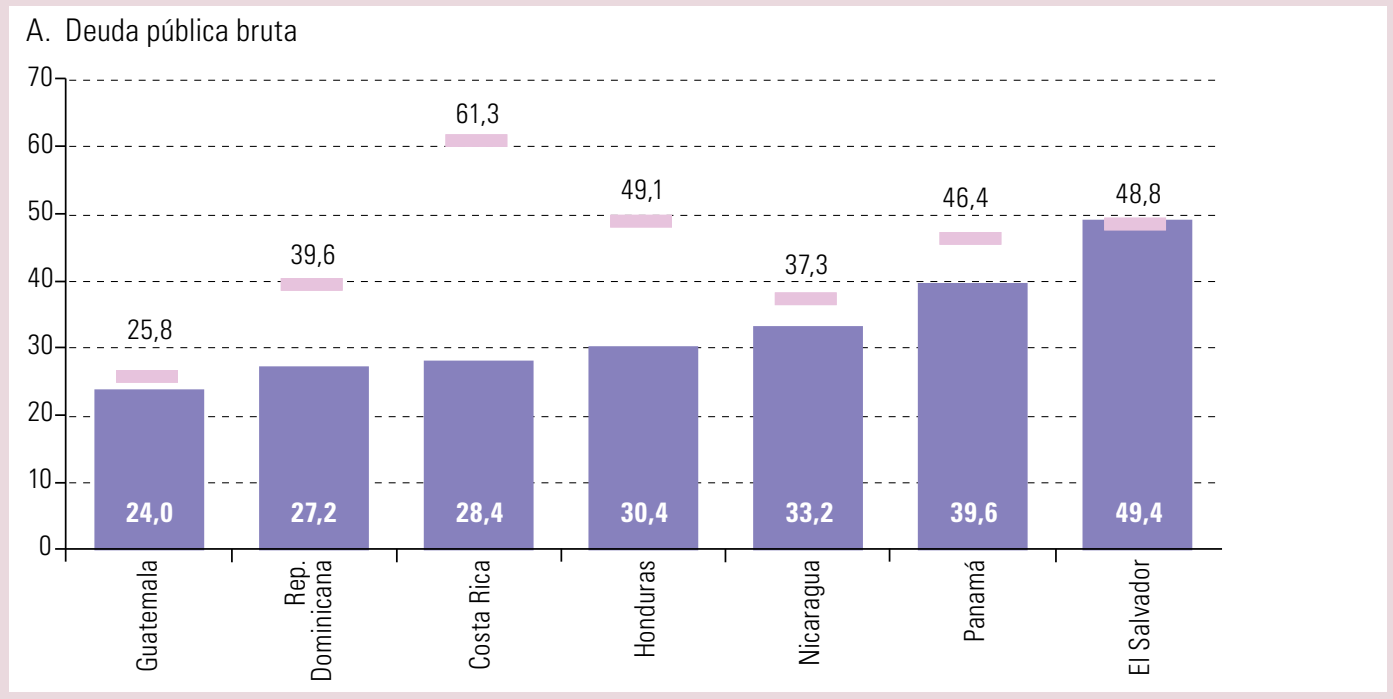

\section{B. Pago de intereses}

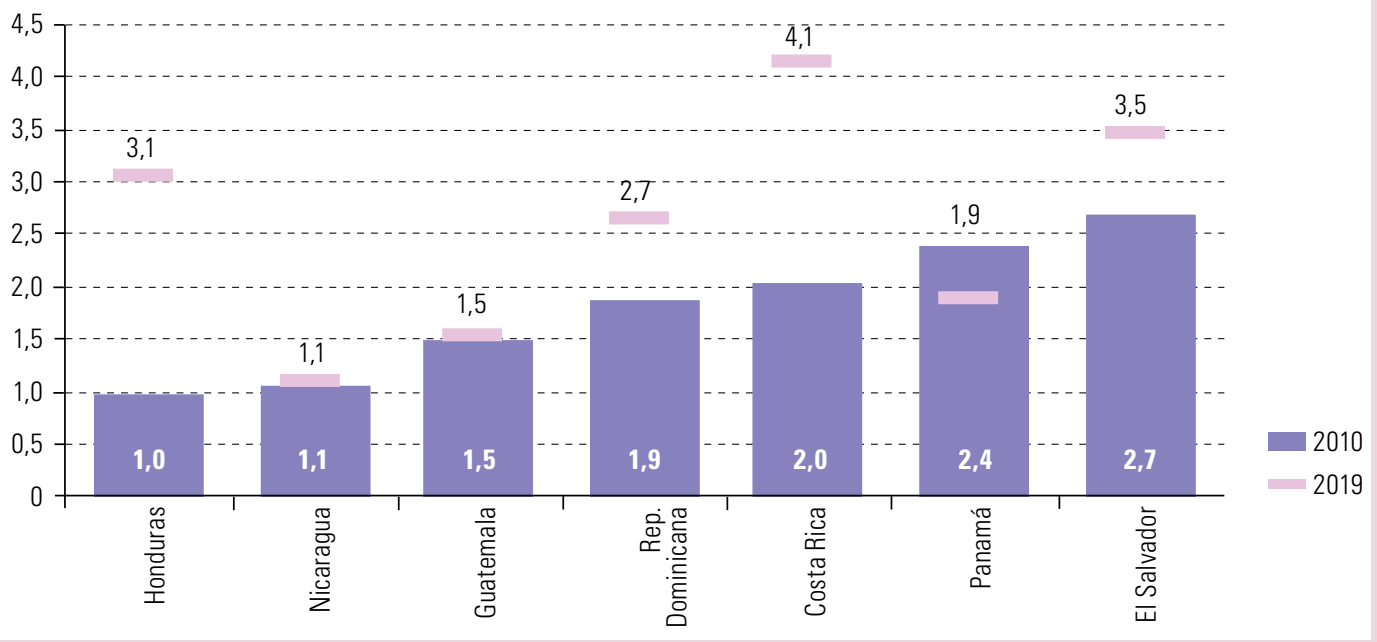

Para mantener la sostenibilidad de la deuda pública, los países de la subregión redujeron los gastos de capital para acomodar el aumento de los pagos de intereses, los que son mayores que el gasto en áreas como la salud, educación y la protección social. El gasto en salud de los gobiernos centrales de El Salvador ( $2,4 \%$ del PIB), Guatemala (1,1\% del PIB), Honduras (2,4\% del PIB), Panamá (1,7\% del PIB) o la República Dominicana $(1,7 \%$ del PIB) es menor que los pagos de intereses de la deuda.

Utilizar las mismas herramientas que en crisis pasadas imposibilitarían orientar recursos a la atención de la emergencia humanitaria, la recuperación de la actividad económica y el mantenimiento de la estabilidad social. Así, los esfuerzos para generar la liquidez y el espacio fiscal deben ser un marco propicio para renovar pactos fiscales y avanzar hacia nuevos contratos sociales.

Un proceso negociado de moratoria de la deuda con las instituciones financieras multilaterales generaría el espacio fiscal y la liquidez imprescindibles para atender la emergencia humanitaria y económica sin desviar las trayectorias y los esfuerzos para asegurar la sostenibilidad de la deuda de la subregión.

Fuente: Comisión Económica para América Latina y el Caribe (CEPAL). 
- Pese a ser considerados en su mayor parte como países de renta media alta, los países del Caribe de habla inglesa, debido a su constante necesidad de financiamiento para recuperar su estructura productiva frente a los desastres climáticos, presentan un alto nivel de endeudamiento público, que se traduce en una fuerte carga de los pagos de intereses en las erogaciones públicas. Aunque el resultado primario de sus gobiernos centrales ha sido superavitario en la mayoría de los años durante la última década (con un promedio del 0,8\% del PIB), los balances globales de los gobiernos centrales se han mantenido deficitarios, con un promedio del 2,5\% del PIB y un máximo del 3,4\% del PIB en 2013.

- La generación de superávits primarios inhibió el financiamiento para la inversión pública y el gasto social. Aunque los altos superávits primarios llevaron a una disminución de la deuda pública de los gobiernos centrales del 72,8\% del PIB en 2017 al 68,5\% del PIB en 2019 (véase el gráfico 4), países como Barbados, Belice y Jamaica aún tienen elevados niveles de deuda (122,6\%, $93,7 \%$ y $87,4 \%$ del PIB, respectivamente) y, por ende, altos pagos de intereses $(3,4 \%, 2,9 \%$ y $6,3 \%$ del PIB, respectivamente). Esto además de su alta vulnerabilidad frente al cambio climático y los desastres naturales que destruyen periódicamente importantes activos económicos.

Gráfico 4 | El Caribe (13 países): niveles de deuda pública bruta de los gobiernos centrales, 2000-2019 (En porcentajes del PIB)

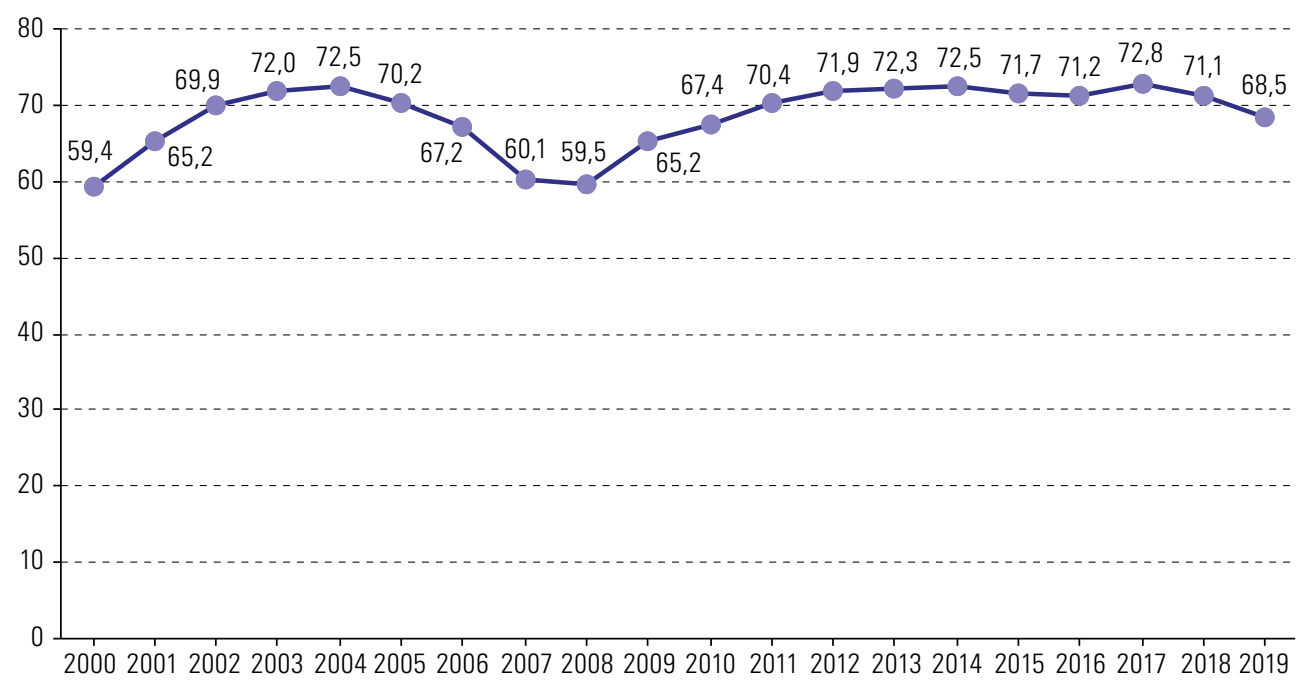

Fuente: Comisión Económica para América Latina y el Caribe (CEPAL), sobre la base de cifras oficiales.

- Para el conjunto para América Latina y el Caribe, el espacio fiscal se ve reducido por los limitados ingresos públicos. Durante la última década, los ingresos públicos totales de los gobiernos centrales de América Latina promediaron el 18,2\% del PIB. Este estancamiento se debió a la ralentización de la actividad económica, la evasión fiscal, la caída de los precios internacionales de las materias primas_que afecta especialmente a América del Sur y a México—, la reducción de aranceles por la apertura comercial y la creciente renuncia fiscal por concesiones a las zonas francas y la maquila. En la coyuntura actual, los ingresos públicos se verán aún más afectados por la fuerte contracción de la actividad económica y los deprimidos precios de las materias primas.

2. Pese a que los esfuerzos de las autoridades monetarias por estimular la demanda agregada han llevado las tasas de política monetaria a niveles históricamente bajos, hasta ahora han sido poco efectivos

- Luego de la normalización de la política monetaria en las economías desarrolladas, en especial en los Estados Unidos, a finales de 2013, y en un contexto de baja inflación, las autoridades monetarias de América Latina y el Caribe enfrentaron el dilema de estimular la demanda agregada o evitar un aumento de la inestabilidad macrofinanciera. En 2019, la prolongada y generalizada desaceleración de la demanda agregada interna en la región, en especial del consumo y la inversión, el consiguiente descenso de la inflación y la menor volatilidad que registraron las monedas de la región posibilitaron la adopción de políticas expansivas con distintos grados de libertad dependiendo del régimen cambiario.

- Para propiciar una reducción de las tasas activas y un aumento del crédito interno, los esfuerzos de las autoridades monetarias se encaminaron a aumentar la liquidez con reducciones de tasas de política monetaria (TPM) y mayores expansiones de los agregados monetarios. 
Estos esfuerzos fueron complementados con intervenciones en los mercados cambiarios para atenuar las presiones sobre las cotizaciones de las divisas y los resultados deficitarios de las cuentas externas. Pese a que las TPM cerraron 2019 en niveles históricamente bajos, las tasas de interés activas se mantuvieron bastante estables y el crédito al sector privado se contrajo en términos reales (véase el gráfico 5). Ambos hechos reflejan la dificultad de que la política monetaria atenúe los efectos del bajo crecimiento sobre el riesgo crediticio y la disposición de los agentes a incrementar sus niveles de consumo y de inversión.

Gráfico 5 | América Latina y el Caribe (países seleccionados): evolución del crédito interno real otorgado al sector privado, promedio de las tasas anualizadas, primer trimestre de 2016 a primer trimestre de 2020 (En porcentajes)

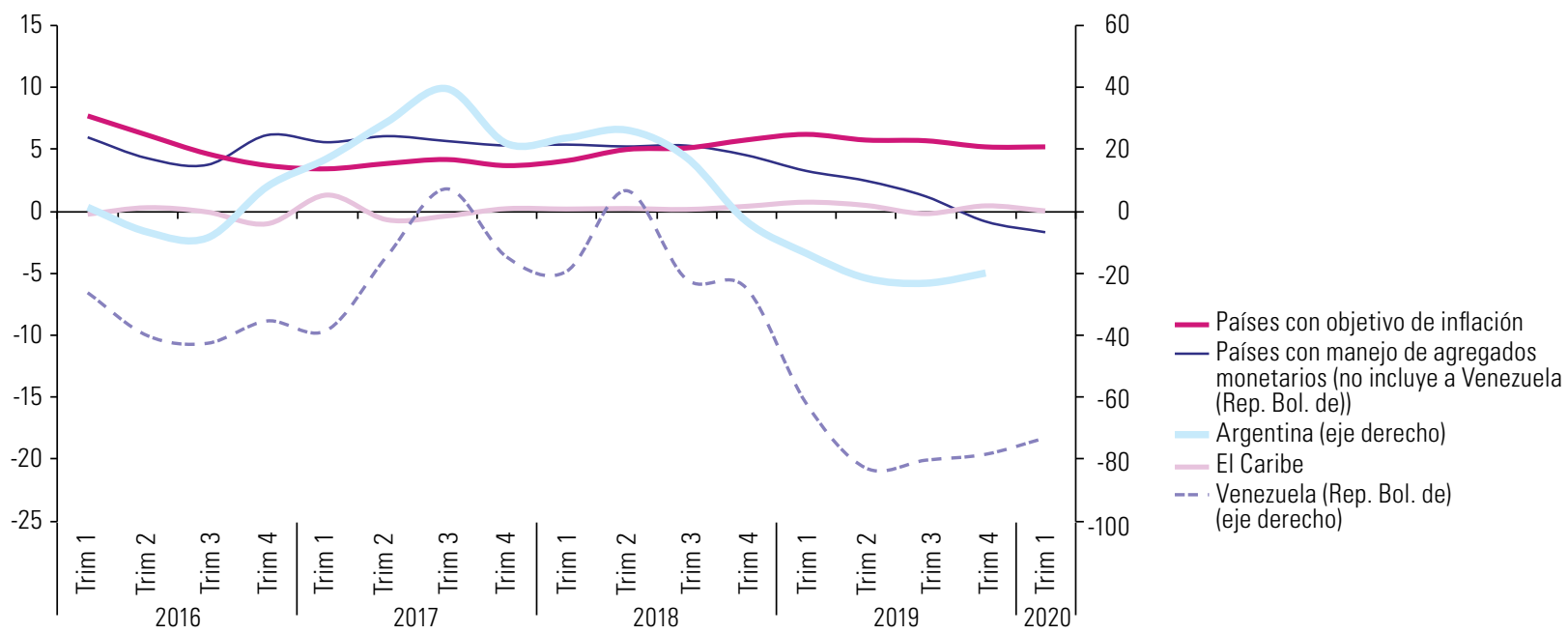

Fuente: Comisión Económica para América Latina y el Caribe (CEPAL), sobre la base de cifras oficiales.

- El elevado nivel de endeudamiento del sector privado (hogares y corporaciones no financieras privadas) ha condicionado la política monetaria y posiblemente su efectividad. El aumento del endeudamiento acompañado con disminuciones de rentabilidad afecta al sector corporativo no financiero en su conjunto y genera preocupación sobre los efectos de la crisis actual en las empresas. Entre el 31 de diciembre de 2001 y el 30 de septiembre de 2019, la deuda privada en la Argentina, el Brasil, Chile, Colombia y México aumentó de 688 a 2.445 millones de dólares (véase el gráfico 6). Además, entre el $47 \%$ y el $62 \%$ de 2.241 empresas de la región en 34 sectores de actividad económica disminuyeron su rentabilidad entre 2010 y 2016.

Gráfico 6 | América Latina (cinco paísesa): evolución de la deuda del sector privado no financiero, datos trimestrales, 2001-2019 (En millones de dólares)

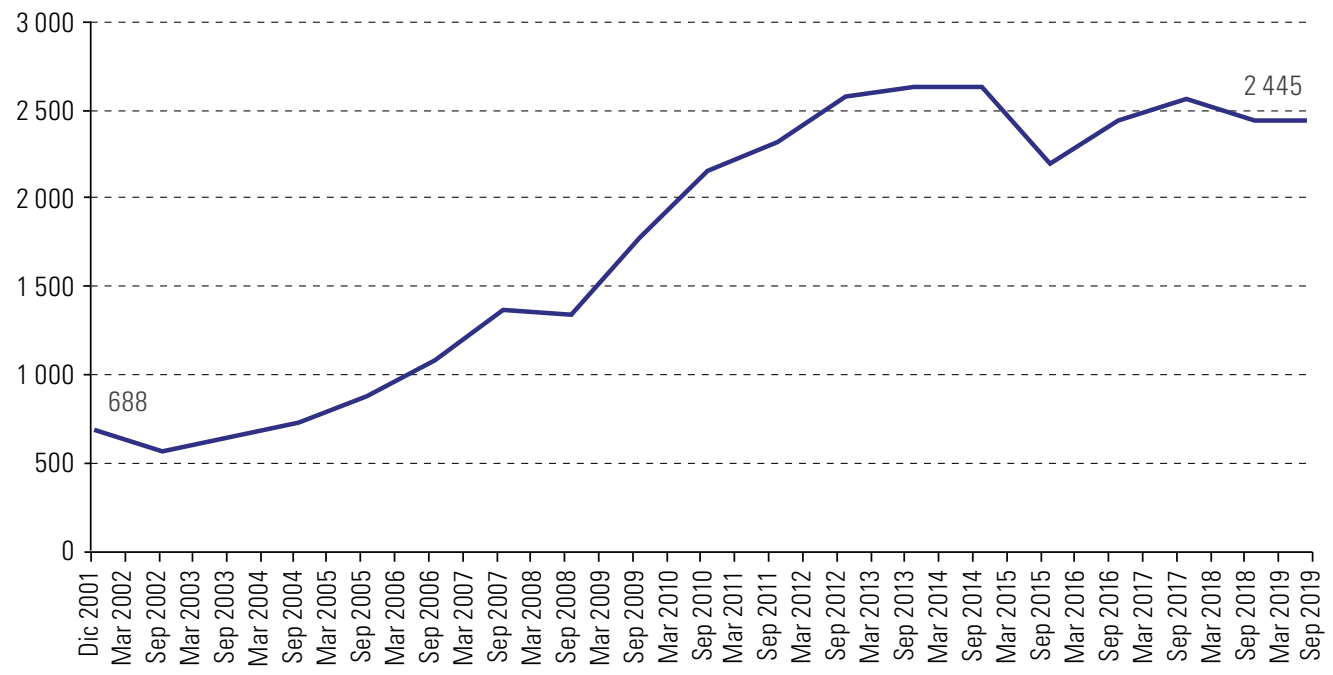

Fuente: Comisión Económica para América Latina y el Caribe (CEPAL), sobre la base de Banco de Pagos Internacionales (BPI), "Debt securities statistics", 2020 [en línea] https://www.bis.org/statistics/secstats.htm.

a Argentina, Brasil, Chile, Colombia y México. 


\section{B. La pandemia llevará a la mayor contracción de la actividad}

económica en la historia de la región

- La pandemia impacta a las economías de América Latina y el Caribe a través de factores externos e internos cuyo efecto conjunto conducirá a la peor contracción de la actividad económica que la región haya sufrido desde que se iniciaron los registros, en 1900.

\section{El choque externo}

- La economía mundial exhibirá en $\mathbf{2 0 2 0}$ una caída del producto bruto mayor a la observada en varias décadas. Se prevé una contracción del PIB mundial en torno al $2 \%$ con una mayor contracción en las economías desarrolladas que en las emergentes.

- En promedio, las proyecciones para los Estados Unidos prevén una caída de casi el $4 \%$ (frente a un crecimiento del 1,9\% pronosticado en diciembre de 2019), para China un crecimiento menor al $\mathbf{2} \%$ (5,8\% pronosticado en diciembre), para la eurozona una caída de casi el $\mathbf{6} \%$ $(1,2 \%$ de crecimiento pronosticado en diciembre) y para el Japón una contracción de más del $\mathbf{4 \%}$ (véase el cuadro 1 ).

Cuadro 1 | Regiones y países seleccionados: tasa de crecimiento del PIB del período 2013-2019 y proyecciones para 2020 (En porcentajes)

\begin{tabular}{lcccccccc}
\hline & $\mathbf{2 0 1 3}$ & $\mathbf{2 0 1 4}$ & $\mathbf{2 0 1 5}$ & $\mathbf{2 0 1 6}$ & $\mathbf{2 0 1 7}$ & $\mathbf{2 0 1 8}$ & $\mathbf{2 0 1 9}$ & $\mathbf{2 0 2 0}$ \\
\hline Mundo & $\mathbf{2 , 6}$ & $\mathbf{2 , 8}$ & $\mathbf{2 , 8}$ & $\mathbf{2 , 6}$ & $\mathbf{3 , 2}$ & $\mathbf{3 , 1}$ & $\mathbf{2 , 4}$ & $\mathbf{- 2 , 0}$ \\
\hline Estados Unidos & 1,8 & 2,5 & 2,9 & 1,6 & 2,4 & 2,9 & 2,3 & $-\mathbf{3 , 8}$ \\
\hline Japón & 2,0 & 0,4 & 1,3 & 0,6 & 2,0 & 0,8 & 0,7 & $-4,2$ \\
\hline Eurozona & $-0,3$ & 1,4 & 2,1 & 1,9 & 2,5 & 1,9 & 1,2 & $-5,7$ \\
\hline China & 7,8 & 7,3 & 6,9 & 6,7 & 6,9 & 6,6 & 6,1 & 1,8 \\
\hline India & 6,4 & 7,4 & 8,0 & 8,2 & 7,2 & 6,8 & 5,8 & 3,4 \\
\hline
\end{tabular}

Fuente: Comisión Económica para América Latina y el Caribe (CEPAL), sobre la base de Instituto de Finanzas Internacionales (IIF), Capital Flows Report: Sudden Stop in Emerging Markets, 9 de abril de 2020; y datos de Bloomberg.

- El volumen de comercio mundial ha colapsado: la Organización Mundial del Comercio (OMC) estima que caerá entre un $13 \%$ y un $32 \%$ en 2020.

- El volumen de comercio mundial de bienes presentaba una tendencia negativa antes de la pandemia. En 2019, disminuyó un 0,4\%, su primera caída desde la crisis financiera mundial (véase el gráfico 7). Esto fue, en gran medida, el resultado de la acumulación de barreras comerciales desde principios de 2018 (principalmente entre los Estados Unidos y China) y su efecto en las cadenas globales de valor. Las perspectivas mejoraron en enero de 2020 luego del acuerdo de "fase uno" entre China y los Estados Unidos, pero la pandemia puso fin a ese corto optimismo.

- A la disrupción de las cadenas de valor, se agrega el hecho de que China, una vez que vaya reactivando su producción, enfrentará problemas para exportar a países que enfrentan la pandemia con un rezago temporal respecto de este país. Así, uno de los principales actores en el comercio mundial verá disminuida la demanda de sus exportaciones, lo que aumentará el impacto negativo sobre el volumen global de comercio.

- La caída de la actividad económica mundial, en particular en los Estados Unidos, China y Europa tiene un impacto negativo en América Latina y el Caribe a través del comercio en términos de volumen y precio, en especial de las materias primas. Algunos importantes sectores productivos de países de la región están insertos en cadenas globales de valor en las que los Estados Unidos y China juegan un papel fundamental. Además, México y Centroamérica están expuestos a la contracción de la economía de los Estados Unidos también a través la reducción de las remesas de los migrantes; en el caso de México se agrega la caída del precio del petróleo. 
Gráfico 7 | Tasa de variación interanual del volumen del comercio mundial de bienes (En porcentajes, sobre la base de un índice desestacionalizado)

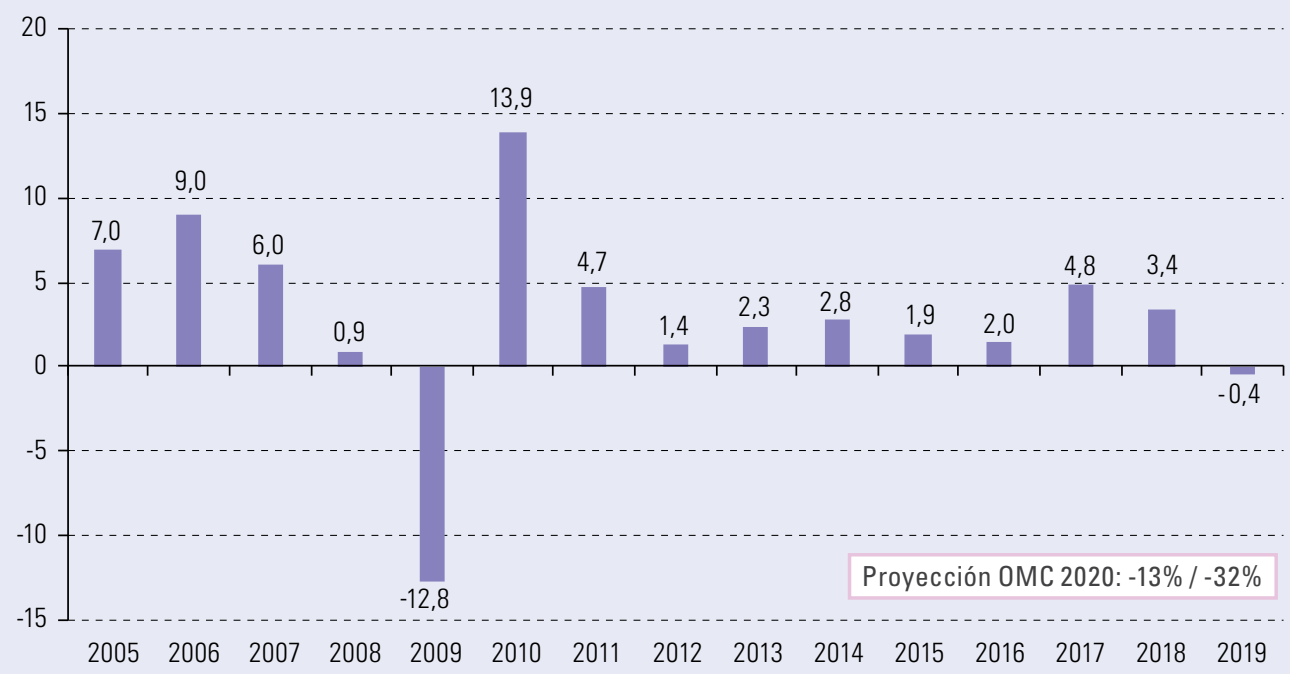

Fuente: Comisión Económica para América Latina y el Caribe (CEPAL), sobre la base de Netherlands Bureau of Economic Policy Analysis (CPB), World Trade Monitor [base de datos en línea] https://www.cpb.nl/en/worldtrademonitor; Organización Mundial del Comercio (OMC), "Trade forecast press conference", 8 de abril de 2020 [en línea] https://www.wto.org/english/news_e/spra_e/spra303_e.htm.

- Ante el nuevo panorama económico en los principales socios de la región y una acentuación del desplome de los precios de exportación, a continuación se revisan resultados presentados en el número 1 de este Informe Especial. La nueva estimación indica que el valor de las exportaciones de la región caería cerca del $\mathbf{1 5 \%}$, con una disminución de los precios del $8,8 \%$ y una contracción del volumen del $6 \%$, explicada principalmente por una agudización de la contracción de la demanda mundial (véase el cuadro 2).

Cuadro 2 | América Latina y el Caribe: efectos del COVID-19 en las exportaciones de bienes por subregiones y países de exportación principales, pronóstico para 2020

(Variación porcentual)

\begin{tabular}{|c|c|c|c|c|c|c|}
\hline \multirow{2}{*}{ Región/Subregión/País } & \multicolumn{3}{|c|}{ Primer Informe Especial COVID-19a } & \multicolumn{3}{|c|}{ Proyecciones actualizadas $^{b}$} \\
\hline & Volumen & Precio & Valor & Volumen & Precio & Valor \\
\hline América Latina y el Caribe & $-2,5$ & $-8,2$ & $-10,7$ & $-6,0$ & $-8,8$ & $-14,8$ \\
\hline Exportadores de petróleo & $-1,8$ & $-14,1$ & $-15,9$ & $-4,7$ & $-14,6$ & $-19,2$ \\
\hline Exportadores de minerales & $-3,0$ & $-8,9$ & $-12,0$ & $-7,4$ & $-9,3$ & $-16,7$ \\
\hline $\begin{array}{l}\text { Exportadores de productos } \\
\text { agroindustriales }\end{array}$ & $-2,4$ & $-2,5$ & $-5,0$ & $-6,2$ & $-4,0$ & $-10,2$ \\
\hline América del Sur & $-2,8$ & $-11,0$ & $-13,8$ & $-6,0$ & $-11,6$ & $-17,6$ \\
\hline Brasil & $-3,7$ & $-7,5$ & $-11,2$ & $-7,0$ & $-8,1$ & $-15,1$ \\
\hline México & $-2,2$ & $-5,2$ & $-7,4$ & $-6,0$ & $-5,7$ & $-11,6$ \\
\hline Centroamérica & $-1,3$ & $-2,7$ & $-4,0$ & $-4,9$ & $-5,3$ & $-10,3$ \\
\hline Países del Caribe & $-2,0$ & $-7,2$ & $-9,3$ & $-6,2$ & $-7,7$ & $-13,9$ \\
\hline
\end{tabular}

Fuente: Comisión Económica para América Latina y el Caribe (CEPAL).

a Se asumen las siguientes tasas de crecimiento para 2020: 1,0\% (mundial), 1,0\% (Estados Unidos), 0,3\% (Japón), 0,5\% (Unión Europea, 27 países), $3,0 \%$ (China) y -1,8\% (América Latina y el Caribe), más una reducción media del 16\% en la cesta de exportación de productos primarios de la región.

${ }^{b}$ Se asumen las siguientes tasas de crecimiento para 2020: -2,0\% (mundial), -3,8\% (Estados Unidos), -4,2\% (Japón), -5,7\% (Unión Europea, 27 países), 1,8\% (China) y -5,3\% (América Latina y el Caribe), más una reducción media del 18\% en la cesta de exportación de productos primarios de la región.

- Se prevé que, en promedio, los bajos precios se prolonguen en el tiempo, incluso para productos que no habían experimentado bajas. Por ejemplo, las cotizaciones de los precios de los productos agrícolas que no habían caído tanto como el petróleo y los metales en las últimas semanas han comenzado a disminuir, debido a la contracción de la demanda. Los precios de la soja, el maíz y el trigo experimentaron disminuciones de hasta un $4 \%$, y los futuros para el segundo trimestre han comenzado a deteriorarse. Similares comportamientos se dan en la carne de bovino y de pollo, con caídas promedio del $6 \%$ en el último bimestre. En el caso del 
petróleo, principal producto de exportación de la región, la fuerte baja de la demanda, estimada en un 30\%, no podrá ser compensada por el último acuerdo de la Organización de Países Exportadores de Petróleo (OPEP) que recorta en un $20 \%$ la producción de los países miembros de la Organización (hasta 9,7 millones de barriles diarios).

- Los mayores impactos se darían en los países de América del Sur, que se especializan en la exportación de bienes primarios y, por lo tanto, son más vulnerables a la disminución de sus precios.

- Las exportaciones regionales a China serían las que más disminuirían en 2020 (24,4\%) (véase el cuadro 3). Esto afectaría especialmente a los productos con eslabonamientos hacia adelante en las cadenas de valor en ese país (mineral de hierro, mineral de cobre, zinc, aluminio, soja, aceite de soja, entre otros). Los países más expuestos son la Argentina, el Brasil, Chile y el Perú, los mayores exportadores de la región de esos productos a China.

Cuadro 3 | América Latina y el Caribe: efectos del COVID-19 en las exportaciones de bienes al mundo y a socios seleccionados, pronóstico para 2020

(Variación porcentual)

\begin{tabular}{lccl}
\hline Destinos & $\begin{array}{c}\text { Proyecciones del primer } \\
\text { Informe Especial COVID-19a }\end{array}$ & $\begin{array}{c}\text { Proyecciones } \\
\text { actualizadas }^{b}\end{array}$ & Sectores y países más afectados \\
\hline Mundo & $-10,7$ & $-14,8$ & \\
\hline China & $-21,7$ & $-24,4$ & $\begin{array}{l}\text { Productos agrícolas y agroindustriales } \\
\text { (Argentina, Brasil, Uruguay, Paraguay); } \\
\text { Productos mineros (Chile y Perú) }\end{array}$ \\
\hline Estados Unidos & $-7,1$ & $-11,6$ & \begin{tabular}{l} 
Manufacturas (México, Costa Rica) \\
\hline Unión Europea
\end{tabular} \\
& $-8,9$ & $-16,1$ & $\begin{array}{l}\text { Productos mineros (Chile, Colombia, } \\
\text { Ecuador, Perú); Productos agrícolas } \\
\text { yagroindustriales (Argentina, Brasil, } \\
\text { Chile, Perú) }\end{array}$ \\
\hline $\begin{array}{l}\text { América Latina } \\
\text { y el Caribe }\end{array}$ & $-10,7$ & $-14,5$ & $\begin{array}{l}\text { Manufacturas de productos de } \\
\text { tecnología baja y media }\end{array}$ \\
\hline
\end{tabular}

Fuente: Comisión Económica para América Latina y el Caribe (CEPAL).

a Se asumen las siguientes tasas de crecimiento para 2020: 1,0\% (mundial), 1,0\% (Estados Unidos), 0,3\% (Japón), 0,5\% (Unión Europea, 27 países), $3,0 \%$ (China) y -1,8\% (América Latina y el Caribe), más una reducción media del 16\% en la cesta de exportación de productos primarios de la región.

b Se asumen las siguientes tasas de crecimiento para 2020:-2,0\% (mundial), -3,8\% (Estados Unidos), -4,2\% (Japón), -5,7\% (Unión Europea, 27 países), 1,8\% (China) y -5,3\% (América Latina y el Caribe), más una reducción media del 18\% en la cesta de exportación de productos primarios de la región.

- Las condiciones financieras a nivel mundial sufrieron un deterioro comparable -incluso mayor en algunos indicadores - al evidenciado durante la crisis financiera mundial de 2008-2009.

- La volatilidad aumentó a niveles históricos y se produjeron salidas masivas de capitales desde los mercados emergentes, una depreciación de la mayor parte de las monedas respecto del dólar y fuertes aumentos en los niveles de riesgo soberano, excepto en los países considerados como refugio a los que "huyen" los inversores en busca de seguridad, y en los que, por el contrario, alcanzaron mínimos históricos. Los mercados bursátiles de todo el mundo experimentaron fuertes caídas reflejo de la preocupación por los efectos de la pandemia en la situación financiera de las empresas.

- El hecho de que los mercados financieros se hayan visto gravemente afectados por el efecto de la pandemia mundial es producto también de vulnerabilidades financieras que se habían ido acumulando durante mucho tiempo. La acumulación de deuda ha estado superando el crecimiento del ingreso mundial y ha alcanzado niveles récord. En el cuarto trimestre de 2019, el acervo de deuda mundial superó los 255 billones de dólares, más del 322\% del PIB mundial (IIF, 2020b).

- El aumento de la deuda fue acompañado por estándares de préstamos más laxos y mayor aceptación de riesgo por parte de los inversores en busca de rendimiento. Si bien el aumento de la deuda se produjo en todos los sectores (hogares, sector corporativo no financiero, gobierno y sector financiero), lo que genera serias preocupaciones ahora es que gran parte de la 
acumulación de deuda desde la crisis financiera mundial se ha dado en sector corporativo no financiero, donde la interrupción de las cadenas de suministro y la reducción del crecimiento global implican menores ganancias y una mayor dificultad en el servicio de la deuda. La recesión afecta la capacidad de los prestatarios corporativos de obtener ganancias y pagar deudas, lo que se agrava por el aumento en los costos de endeudamiento y las interrupciones en la capacidad de financiamiento que enfrentan esos prestatarios.

- En América Latina, la menor disponibilidad de flujos de financiación hacía economías emergentes se traduce en una ralentización de los flujos de capitales del exterior (una disminución de casi 80.000 millones de dólares respecto de 2019) y salidas netas en los flujos de cartera y otra inversión por parte de no residentes (véase el gráfico 8) (IIF, 2020c).

Gráfico 8 | Flujos de capitales de no residentes hacia América Latina, 2000-2020

(En miles de millones de dólares)

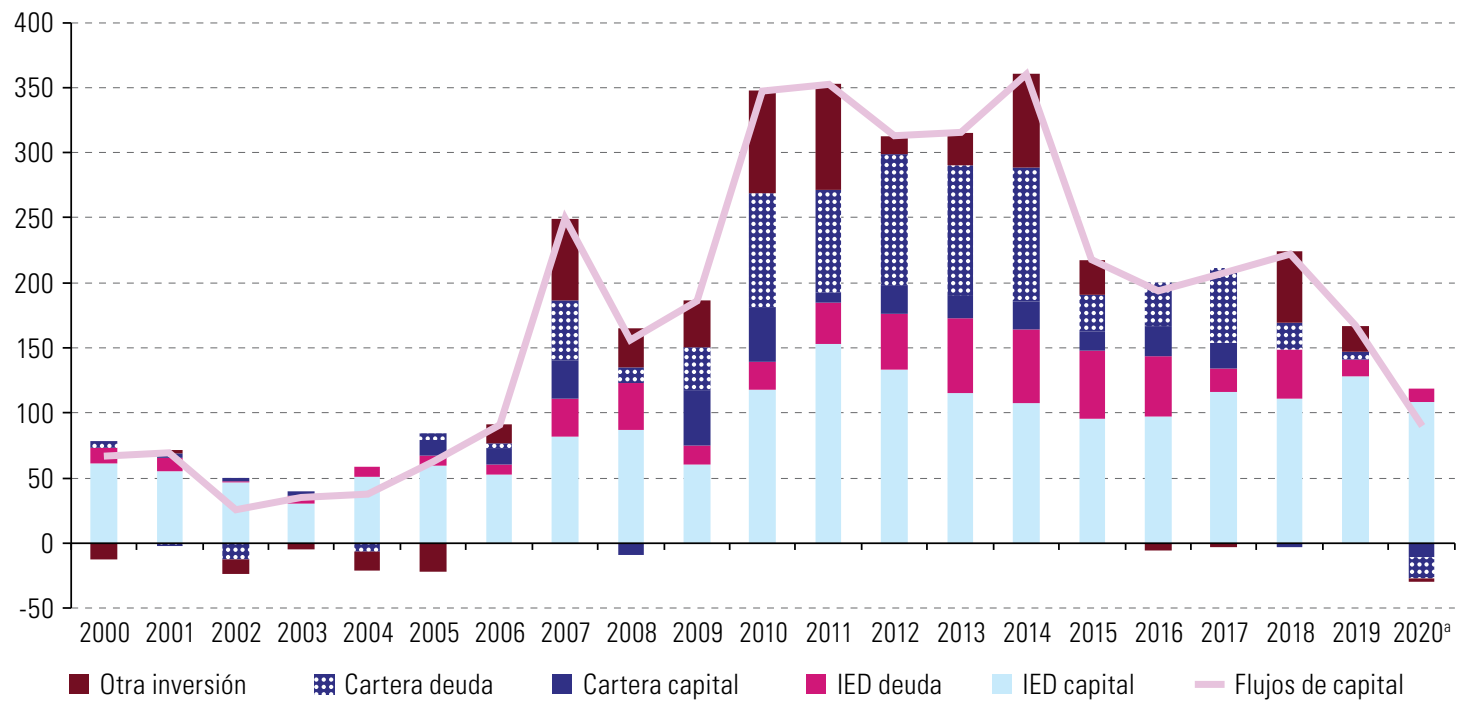

Fuente: Instituto de Finanzas Internacionales (IIF).

a Proyección.

- Los flujos de remesas hacia América Latina y el Caribe se podrían contraer entre un $10 \%$ y un 15\% en 2020 y podrían pasar entre 4 y 8 años para que retomen el monto alcanzado en 2019. En varios países de la región, la contribución de este flujo a la actividad económica es muy significativa (véase el gráfico 9). En Haití representaron más del 30\% del PIB; El Salvador y Honduras aportaron en torno al $20 \%$, y en Jamaica, Guatemala y Nicaragua su peso fue superior al $10 \%$. Entre un $80 \%$ y un $90 \%$ de las remesas se usan para cubrir necesidades básicas de los hogares receptores (alimentación, salud y vivienda), por lo que su contracción tendrá fuertes efectos en el consumo y la incidencia de la pobreza.

- En el principal país de destino de los migrantes de la región (Estados Unidos), la crisis sanitaria afecta a sectores en los que dichos migrantes tradicionalmente se emplean, como la construcción, los restaurantes y los hoteles. En 2018, el 28,7\% de los migrantes provenientes de los países de la región se empleaban en servicios y el 20,6\% en construcción y mantenimiento (Oficina del Censo de los Estados Unidos, 2018). El impacto de la crisis en las remesas podría verse atenuado por los apoyos directos (transferencias) que reciban los migrantes en los lugares de destino y las familias receptoras en las comunidades de origen. La crisis también ha mostrado los aportes de la fuerza laboral inmigrante en sectores como la producción de alimentos y los servicios de salud en estados donde tienen una presencia considerable.

- El turismo es uno de los sectores más afectados y su recuperación depende de la apertura de las fronteras a nivel mundial. En 2020 se registraría una reducción de entre un $20 \%$ y un $30 \%$ en el número de llegadas de turistas en el mundo, una caída mucho mayor que la observada en 2009 (4\%) (OMT, 2020). En un escenario en el que los ingresos por turismo disminuyeran un $30 \%$ en 2020, el PIB se reduciría 2,5, 0,8 y 0,3 puntos porcentuales en el Caribe, México y Centroamérica, y América del Sur, respectivamente. Las repercusiones en el empleo, los 
ingresos de los hogares y los ingresos gubernamentales serían mayores en el Caribe, donde ese sector emplea a unos 2,4 millones de personas y representa el 15,5\% del PIB (véase el gráfico 10). Los efectos de la retracción del turismo se sentirán en particular a las micro y pequeñas empresas, cuyo peso en el sector de hoteles y restaurantes es enorme: el 99\% de las empresas y el 77\% del empleo (CEPAL, 2020).

Gráfico 9 | América Latina y el Caribe: flujos de remesas familiares como porcentaje del PIB, 2018 (En porcentajes)

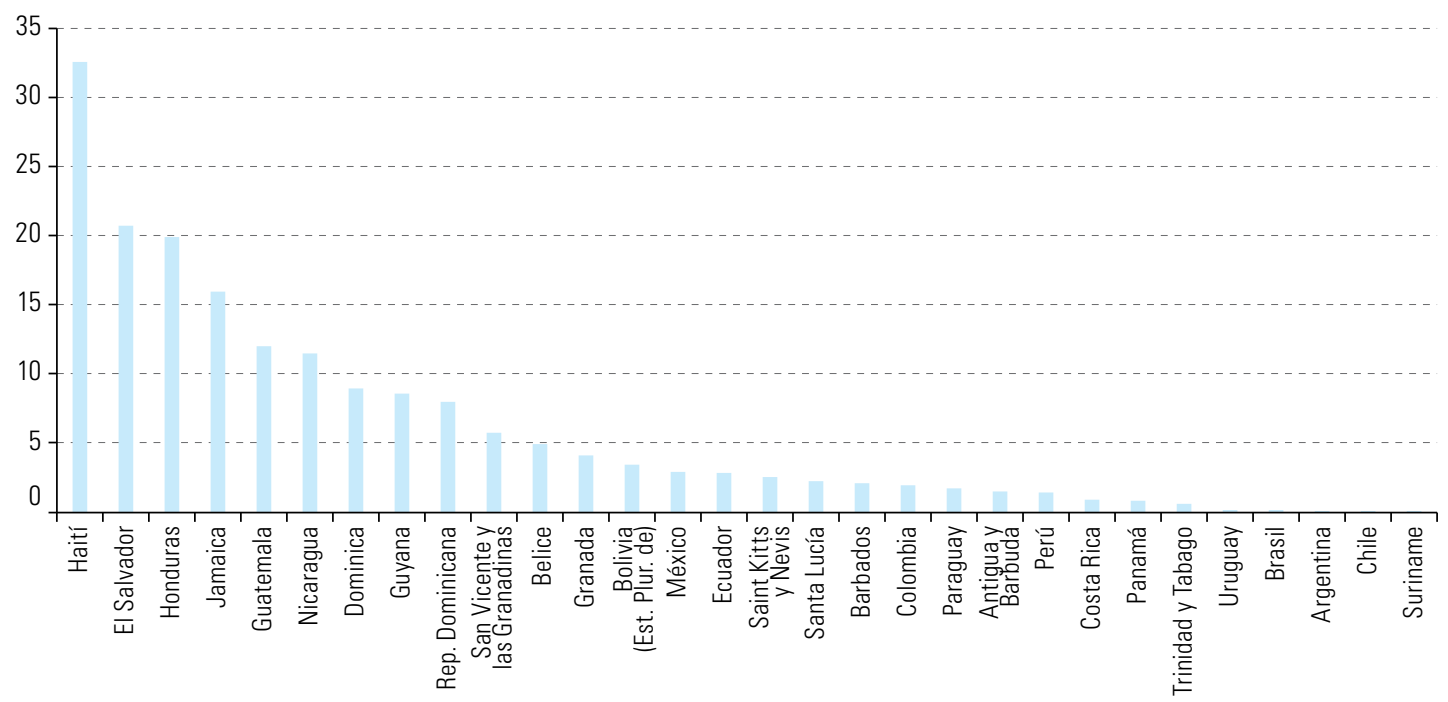

Fuente:Comisión Económica para América Latina y el Caribe (CEPAL), sobre la base de Banco Mundial, World Development Indicators (WDI) [base de datos en línea] http://datatopics.worldbank.org/world-development-indicators/.

Gráfico 10 | América Latina y el Caribe: contribución del turismo al PIB y el empleo, 2018 (En porcentajes)

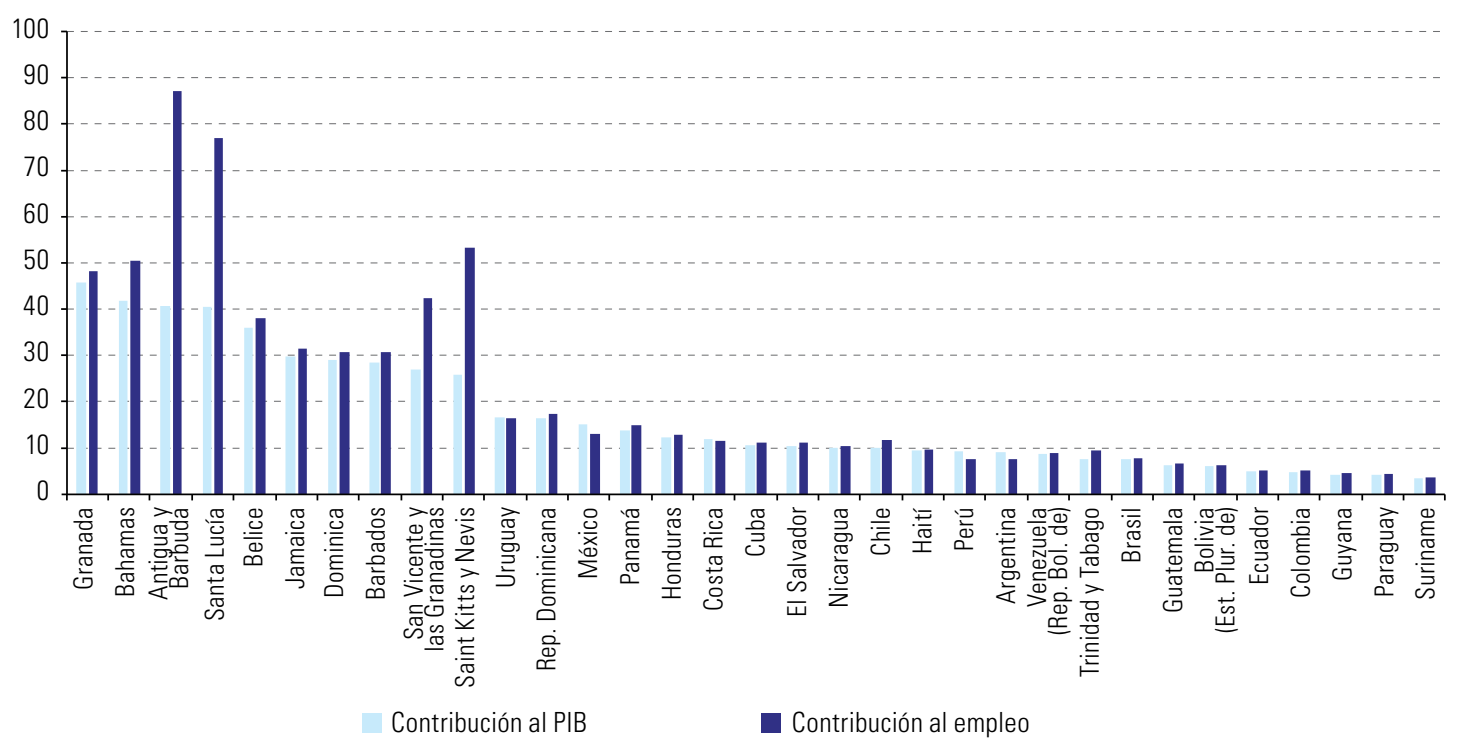

Fuente: Comisión Económica para América Latina y el Caribe (CEPAL), sobre la base de datos del Consejo Mundial de Viajes y Turismo.

\section{El choque interno}

- Se agregan fuertes choques internos al choque externo en la medida que las políticas de contención sanitaria para prevenir la propagación de la pandemia conducen a una paralización de la producción y actividad económica. Ante la inexistencia de tratamientos y vacunas para enfrentar al virus, los esfuerzos para controlar la epidemia se centran en restricciones a la circulación de individuos, lo que afecta la actividad productiva con diferente intensidad según los sectores. Las cuarentenas y el aislamiento social implicaron marcadas caídas en actividades como la aviación, el turismo, el comercio y las zonas francas. 
- Las empresas, independientemente de su tamaño, enfrentan importantes disminuciones de sus ingresos, dificultades para el acceso al crédito y un aumento de la probabilidad de insolvencia.

- Además, los trabajadores sufren un fuerte impacto en las condiciones laborales, empleo o ingresos, que sumado a lo anterior lleva a un deterioro de segunda vuelta en la demanda agregada de los países y a aumentos en la pobreza.

- De acuerdo con la intensidad con que se verían afectados por las medidas de contención, hay tres grupos de sectores con pesos muy diferentes en el PIB según los países; estos pesos se estiman de forma directa sin considerar impactos secundarios o intersectoriales (véase el cuadro 4):

i) Los menos afectados: agricultura, ganadería, caza, silvicultura y pesca.

ii) Los medianamente afectados: explotación de minas y canteras, industrias manufactureras, suministro de electricidad, gas y agua, construcción, intermediación financiera, inmobiliarias, servicios empresariales y de alquiler, administración pública, servicios sociales y personales.

iii) Los más afectados: comercio al por mayor y al por menor, reparación de bienes, hoteles y restaurantes, transporte, almacenamiento y comunicaciones, servicios en general.

Cuadro 4 | América Latina: participación en el PIB de los sectores productivos según intensidad del impacto del COVID-19

(En porcentajes)

\begin{tabular}{lcccc}
\hline & \multicolumn{3}{c}{ Sectores } & \\
\cline { 2 - 5 } & Menos afectados & Medianamente afectados & Más afectados & Total \\
\hline Argentina & 10,5 & 63,6 & 25,9 & 100,0 \\
\hline $\begin{array}{l}\text { Bolivia (Estado } \\
\text { Plurinacional de) }\end{array}$ & 14,4 & 65,0 & 20,6 & 100,0 \\
\hline Brasil & 7,9 & & 16,4 & 100,0 \\
\hline Chile & 3,7 & 75,8 & 21,4 & 100,0 \\
\hline Colombia & 6,8 & 75,0 & 22,2 & 100,0 \\
\hline Costa Rica & 5,3 & 71,0 & 23,0 & 100,0 \\
\hline Ecuador & 9,9 & 71,7 & 23,6 & 100,0 \\
\hline Guatemala & 10,5 & 66,5 & 30,4 & 100,0 \\
\hline El Salvador & 10,4 & 59,1 & 24,1 & 100,0 \\
\hline Honduras & 12,1 & 65,5 & 24,3 & 100,0 \\
\hline México & 3,4 & 63,6 & 30,7 & 100,0 \\
\hline Nicaragua & 16,2 & 65,9 & 23,9 & 100,0 \\
\hline Panamá & 2,4 & 59,9 & 35,5 & 100,0 \\
\hline Perú & 6,3 & 62,1 & 26,3 & 100,0 \\
\hline Paraguay & 12,1 & 67,4 & 0,0 & 100,0 \\
\hline Uruguay & 6,7 & 87,9 & 38,5 & 100,0 \\
\hline Venezuela(República & 5,1 & 54,8 & 22,4 & 100,0 \\
\hline Bolivariana de) & & 72,5 & & \\
\hline
\end{tabular}

Fuente: Comisión Económica para América Latina y el Caribe (CEPAL), sobre la base de cifras oficiales.

Nota: Cálculos sobre la base del nivel de PIB en términos reales de 2019.

- Esta tipología de sectores, que será usada más adelante para estimar los efectos de la pandemia sobre la evolución del PIB en 2020, tiene diversas dimensiones en términos de dinamismo, estructura productiva e intensidad tecnológica, que se analizarán en números siguientes de este Informe Especial. 


\section{Respuestas de política para enfrentar los efectos económicos y sociales de la pandemia}

- Los países de América Latina y el Caribe han tomado rápidas e importantes medidas para responder a la etapa inicial de la crisis mediante la adopción de conjuntos amplios de políticas económicas para mitigar sus efectos sociales y económicos. Esas medidas se enfocan en incrementar la disponibilidad de recursos públicos para el sector de la salud, proteger a los hogares -especialmente los más vulnerables_, proteger la capacidad productiva y el empleo, evitar una crisis de liquidez y evitar el colapso del sistema económico (véase el cuadro 5).

Cuadro 5 | Medidas anunciadas por los países para enfrentar la pandemia

Los países de la región han implementado medidas inmediatas para contener el virus, proteger la fuerza laboral y los ingresos de los hogares

- Medidas de restricción y monitoreo de viajeros de zonas afectadas por el COVID-19.

- Prohibiciones de vuelo (desde y hacia países específicos) y cierre de fronteras.

- Aprendizaje a distancia y educación en el hogar (con asimetrías en la conectividad).

- Distanciamiento social con cierre de restaurantes, bares, cines, promoción del teletrabajo y reducción del horario de oficina.

- Fortalecimiento de la capacidad del sector de la salud en cuanto a camas, hospitales móviles y suministros médicos; la infraestructura de salud en la región está fragmentada y es insuficiente (poco más de dos camas por cada 1.000 personas).
- Cuarentenas totales o parciales con acuerdos laborales que incluyen medidas legales para teletrabajo, reducción de horas de trabajo, introducción de horarios flexibles, vacaciones adelantadas y protección a los trabajadores de los despidos.

- Protección de la fuerza laboral y de los ingresos mediante apoyo salarial para compensar pérdidas de ingresos con pagos adelantados, subsidios salariales o a través del fortalecimiento del seguro de desempleo, suspensión o reducción de los pagos de contribuciones de servicios de agua, gas y electricidad o a fondos de pensiones privados.

- Protección social para reducir el impacto social en los más pobres mediante subsidios, transferencias de efectivo o pagos adelantados, programas sociales y mecanismos de protección social.

- Apoyo financiero especial para pymes, otras empresas y el sector informal mediante la renegociación y la mejora de las condiciones de crédito bancario.

Medidas monetarias, fiscales y sociales para evitar el colapso del sistema económico

- Medidas monetarias y financieras para preservar la liquidez de los bancos, apoyar el comercio y abordar deudas públicas y privadas; reducción de tasas de interés de los bancos centrales y apertura de líneas de crédito especiales, emisión de préstamos adicionales, ampliación de los plazos para el reembolso de los créditos (consumidores y empresas) o extensión de hipotecas.

- Medidas fiscales que incluyen la flexibilidad para redirigir presupuestos estatales, proceder a la desgravación fiscal, retrasar los plazos de presentación de declaraciones de impuestos o exenciones de impuestos para ciertos sectores económicos; los ministros de finanzas deberán reorganizar los presupuestos y buscar financiamiento adicional para canalizar recursos hacia medidas urgentes a corto plazo. La Argentina, Bahamas, Barbados, Belice, el Brasil, Chile, Colombia, Guatemala, Honduras, Jamaica, el Paraguay, el Perú, Trinidad y Tabago y el Uruguay han implementado paquetes fiscales que van del 0,6\% a más del $10 \%$ del PIB si se incluyen las garantías estatales a créditos.

- Medidas para preservar la capacidad productiva y crear condiciones para la reactivación de la actividad económica a través de mecanismos de liquidez para empresas, en particular para las pymes.

- Medidas de expansión de los sistemas de protección social para llegar a trabajadores subempleados o autónomos, jóvenes, mujeres, niños, niñas y personas mayores.

Fuente: Comisión Económica para América Latina y el Caribe (CEPAL).

- La magnitud de los esfuerzos en política fiscal es mayor a los impulsos aplicados durante la crisis financiera mundial. Algunos casos destacan en la región; por ejemplo, el Perú ha anunciado medidas cuyo costo representa poco más del 7\% del PIB y son complementadas por garantías estatales de crédito que llegan a un $4 \%$ del PIB, al tiempo que Chile ha anunciado acciones por montos equivalente al 5,5\% del PIB, además de garantías estatales para créditos que podrían llegar hasta el 10\% del PIB.

- Inicialmente los países han tomado acciones para liberar recursos rápidamente a través del uso generalizado de reasignaciones presupuestarias para fortalecer sistemas de salud y 
ampliar las redes de protección social con transferencias que permitan compensar las pérdidas de ingresos de los empleados formales e informales. Estos esfuerzos a menudo han sido acompañados de medidas de alivio tributario para reducir la presión sobre el flujo de caja de las empresas. Adicionalmente, se han extendido garantías de crédito para asegurar que empresas, particularmente las pequeñas y medianas, tengan acceso a liquidez sujeto a que mantengan sus niveles de empleo.

- Por su parte, las autoridades monetarias han tomado medidas para expandir la liquidez, recurriendo al uso de sus instrumentos "tradicionales" y han bajado las tasas de política monetaria (véase el gráfico 11) y las tasas de encaje legal bancario. Han anunciado la creación de líneas de asistencia para las instituciones financieras, especialmente a través de los mercados de corto plazo, para evitar situaciones de descalce e impedir que situaciones de iliquidez se transformen en situaciones de insolvencia. También se han intensificado las intervenciones en el mercado cambiario, no para revertir las depreciaciones que se han dado, sino para atenuar la volatilidad.

- Las medidas de estímulo fiscal ante la crisis humanitaria y económica resultarán en un aumento considerable de los déficits fiscales y los niveles de deuda de muchos países. Por ello es necesario renovar los pactos fiscales en muchos países de la región para enfrentar futuros ajustes y minimizar los riesgos financieros.

Gráfico 11 América Latina (países seleccionados): tasa de política monetaria en los países que la emplean como principal instrumento de política, enero de 2016 a abril de 2020 (En porcentajes)
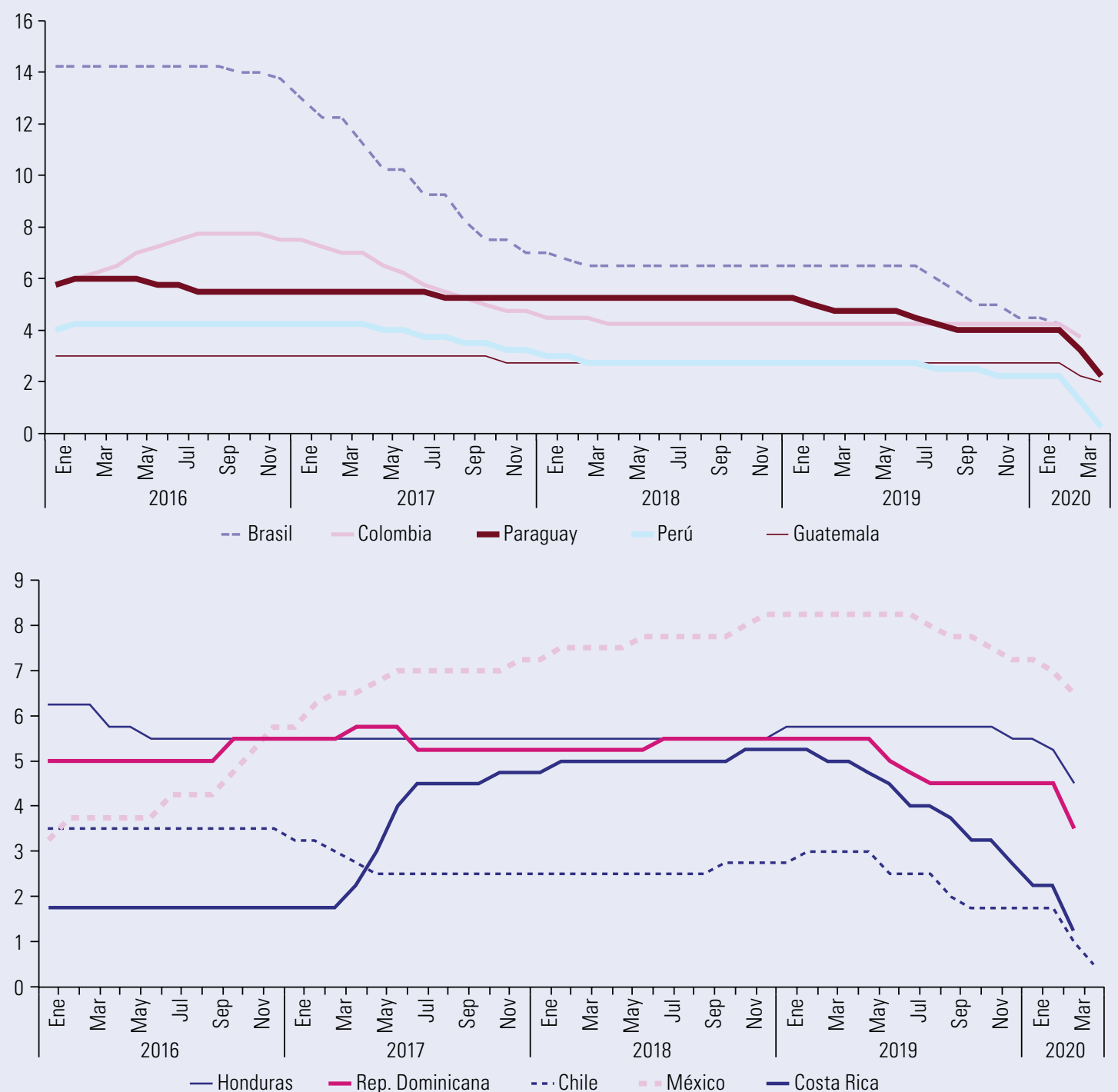

Fuente: Comisión Económica para América Latina y el Caribe (CEPAL), sobre la base de cifras oficiales. 


\section{Proyecciones de crecimiento de los países de América Latina}

y el Caribe en 2020

\section{Metodología}

- Para las estimaciones de crecimiento, en primera instancia se estimó el efecto de la caída en el crecimiento global sobre las economías de la región. Para ello, se utilizó como aproximación el impacto de un menor crecimiento en los Estados Unidos y China. Las elasticidades se calcularon en base a información del FMI (2019).

- En segunda instancia se estimó el impacto del choque de oferta interno de las medidas de cuarentena y aislamiento social que llevan a la paralización en distintos grados de los sectores de actividad económica, según la clasificación en tres grupos de sectores de acuerdo a la intensidad en que serían afectados por las medidas de contención (véase la sección B.2).

- Para la proyección, se supuso que la actividad productiva comenzó a verse afectada en la segunda quincena del mes de marzo. Los grados de afectación se determinaron de forma mensual para cada país a partir de los momentos de establecimiento de las cuarentenas y otras medidas. En la dinámica de las proyecciones, el supuesto - extremadamente incierto- es que la evolución de la pandemia permitirá a las autoridades una flexibilización gradual de las medidas de contención sanitaria a partir del tercer trimestre del año y, con ella, la recuperación gradual de la producción.

\section{La CEPAL proyecta que la economía de América Latina y el Caribe sufrirá} una contracción de la actividad del 5,3\% en 2020, lo que generará casi 30 millones más de pobres

- Para América del Sur, se prevé una caída del 5,2\%. Algunos países de esta subregión son muy afectados por la caída de la actividad en China, que es un importante mercado para sus exportaciones de bienes. Tal es el caso de Chile, el Brasil, el Perú y el Uruguay que destinan a China más del $20 \%$ de sus exportaciones (más del 30\% en el caso de Chile). América del Sur se verá afectada también por la baja en los precios de los productos básicos.

- La interrupción de las cadenas de valor impactará con mayor intensidad en las economías brasileña y mexicana, cuyos sectores manufactureros son los mayores de la región. Para el Brasil, se prevé una caída del $5.2 \%$ y para México una caída del 6,5\%.

- Para las economías de Centroamérica, se espera una caída menor (2,3\%). Los menores precios de los productos básicos no serían un problema ya que son importadoras netas de alimentos y energía. Sin embargo, sí tienen fuertes efectos negativos la caída del turismo y la reducción de la actividad de los Estados Unidos, principal socio comercial y la mayor fuente de inversión extranjera directa y remesas de esos países.

- Las economías del Caribe tendrían una caída del 2,6\%, marcadamente impulsada por la reducción de la demanda por servicios turísticos, que son intensivos en trabajo.

- Las proyecciones también anticipan un importante deterioro de los indicadores laborales en 2020.

- La tasa de desempleo se ubicaría en torno al $\mathbf{1 1 , 5 \% , ~ u n ~ a u m e n t o ~ d e ~ 3 , 4 ~ p u n t o s ~ p o r c e n t u a l e s ~}$ respecto al nivel de $2019(8,1 \%)$. Así, el número de desempleados de la región llegaría a 37,7 millones, lo que implicaría un aumento cercano a 11,6 millones con respecto al nivel de 2019 (26,1 millones de desempleados). Estas proyecciones suponen que el número de ocupados caería cerca del 2,4\% con respecto al nivel de 2019. Los países del Caribe sufrirían una caída mayor debido a que, en muchos de ellos, el sector del turismo es fuente de más del $50 \%$ del empleo. Estas cifras son significativamente mayores a las que se dieron durante la crisis financiera mundial, cuando la tasa de desempleo de la región se incrementó del 6,7\% en 2008 al 7,3\% en 2009 (0,6 puntos porcentuales).

- La alta tasa de informalidad laboral de la región hace a los trabajadores muy vulnerables a los efectos de la pandemia y a las medidas para enfrentarla. 
Cuadro 6 | Proyecciones de crecimiento del PIB de América Latina y el Caribe, 2020

\begin{tabular}{|c|c|}
\hline & $\begin{array}{l}\text { Crecimiento del PIB } \\
2020\end{array}$ \\
\hline América Latina y el Caribe & $-5,3 \%$ \\
\hline Argentina & $-6,5 \%$ \\
\hline Bolivia (Estado Plurinacional de) & $-3 \%$ \\
\hline Brasil & $-5,2 \%$ \\
\hline Chile & $-4 \%$ \\
\hline Colombia & $-2,6 \%$ \\
\hline Ecuador & $-6,5 \%$ \\
\hline Paraguay & $-1,5 \%$ \\
\hline Perú & $-4 \%$ \\
\hline Uruguay & $-4 \%$ \\
\hline Venezuela (República Bolivariana de) & $-18 \%$ \\
\hline América del Sur & $-5,2 \%$ \\
\hline Costa Rica & $-3,6 \%$ \\
\hline Cuba & $-3,7 \%$ \\
\hline El Salvador & $-3 \%$ \\
\hline Guatemala & $-1,3 \%$ \\
\hline Haití & $-3,1 \%$ \\
\hline Honduras & $-2,8 \%$ \\
\hline México & $-6,5 \%$ \\
\hline Nicaragua & $-5,9 \%$ \\
\hline Panamá & $-2 \%$ \\
\hline República Dominicana & $0 \%$ \\
\hline Centroamérica y México & $-5,5 \%$ \\
\hline Centroamérica & $-2,3 \%$ \\
\hline América Latina & $-5,3 \%$ \\
\hline Antigua y Barbuda & $-7,2 \%$ \\
\hline Bahamas & $-6,8 \%$ \\
\hline Barbados & $-5,8 \%$ \\
\hline Belice & $-3,9 \%$ \\
\hline Dominica & $-3,6 \%$ \\
\hline Granada & $-7,3 \%$ \\
\hline Guyana & $56,4 \%$ \\
\hline Jamaica & $-5,3 \%$ \\
\hline Saint Kitts y Nevis & $-6,5 \%$ \\
\hline San Vicente y las Granadinas & $-3,6 \%$ \\
\hline Santa Lucía & $-8,1 \%$ \\
\hline Suriname & $-4,4 \%$ \\
\hline Trinidad y Tabago & $-5 \%$ \\
\hline El Caribe & $-2,5 \%$ \\
\hline
\end{tabular}

Fuente: Comisión Económica para América Latina y el Caribe (CEPAL).

Nota: Centroamérica incluye a Cuba, Haití y la República Dominicana.

- La elevada participación de las pequeñas y medianas empresas en la creación del empleo (más del 50\% del empleo formal) aumenta los impactos negativos, pues este sector ha sido duramente afectado por la crisis.

- La desigualdad de género se acentuará con medidas como el cierre de las escuelas, el aislamiento social y el aumento de personas enfermas, pues aumentará la sobrecarga de trabajo no remunerado de las mujeres.

- La caída del 5,3\% del PIB y el aumento del desempleo en 3,4 puntos porcentuales tendrían un efecto negativo directo sobre los ingresos de los hogares y su posibilidad de contar con recursos suficientes para satisfacer las necesidades básicas.

- En ese contexto, la tasa de pobreza aumentaría 4,4 puntos porcentuales durante 2020 , al pasar del $30,3 \%$ al $34,7 \%$, lo que significa un incremento de 28,7 millones de personas en situación de pobreza (véase el cuadro 7). 
Cuadro 7 | América Latina: pobreza y extrema pobreza en un escenario de caída del PIB del 5,3\% y de aumento del desempleo en 3,4 puntos porcentuales en 2020

(En millones de personas y porcentajes)

\begin{tabular}{lccc}
\hline Año & $\mathbf{2 0 1 8}$ & $\mathbf{2 0 1 9}$ & $\mathbf{2 0 2 0 ^ { \mathbf { a } }}$ \\
\hline Población & 607,7 & 613,5 & 619,2 \\
\hline Pobreza & & & \\
\hline América Latina (18 países) & 180,6 & 186,0 & 214,7 \\
\hline Tasa & $29,7 \%$ & $30,3 \%$ & $\mathbf{3 4 , 7 \%}$ \\
\hline Pobreza extrema & & & \\
\hline América Latina (18 países) & 62,5 & 67,5 & $\mathbf{1 3 , 5 \%}$ \\
\hline Tasa & $10,3 \%$ & $11,0 \%$ & \\
\hline
\end{tabular}

Fuente: Comisión Económica para América Latina y el Caribe (CEPAL).

a Estas estimaciones son preliminares y no toman en consideración el impacto diversificado entre los sectores productivos y el empleo generado en cada uno de ellos.

- Por su parte, la pobreza extrema crecería 2,5 puntos porcentuales, pasando del $11,0 \%$ al $13,5 \%$, lo que representa un incremento de 16 millones de personas.

- También se deterioraría la distribución del ingreso. El aumento del desempleo resultaría en un incremento del índice de Gini de entre un $\mathbf{1 \%}$ y un $\mathbf{2 \%}$, sin considerar el aumento adicional que provendría de la una caída en los ingresos del empleo que afecte en mayor medida a los hogares de menores recursos.

\section{E. Desafíos de la política macroeconómica para seguir avanzando en la mitigación de los efectos de la crisis}

- Los paquetes fiscales anunciados en la región son la primera respuesta al impacto socioeconómico de la pandemia. Se requerirán esfuerzos adicionales en la medida que aumente la magnitud de la crisis. Por lo tanto, es necesario expandir el espacio fiscal, lo que requiere acceder a condiciones de financiamiento favorables.

- Un número cada vez mayor de países de la región ha solicitado financiamiento de emergencia a las instituciones financieras internacionales, como el Fondo Monetario Internacional (FMI), el Banco Mundial, el Banco Interamericano de Desarrollo (BID) y el Banco de Desarrollo de América Latina (CAF), las que han ampliado el acceso al crédito a los países miembros. Es importante que estas instituciones continúen facilitando las líneas de crédito a bajo costo y de forma ágil, además de revisar las condicionalidades en el financiamiento de emergencia y flexibilizar las políticas de préstamos concesionales y de graduación de los países de renta media.

- La condonación y el alivio del servicio de la deuda, incluido a los países de ingresos medios de la región que lo requieran, aumentaría significativamente su espacio fiscal. Las economías altamente endeudadas deben beneficiarse de alivios oficiales de la deuda o de suspensiones generosas de sus obligaciones, así como de reestructuraciones de deuda. Abordar la problemática de la deuda es urgente y debe ser liderada por acreedores oficiales para luego atraer a acreedores privados. Las quitas de deuda deberían combinarse con mecanismos innovadores de financiamiento cuando la deuda sea una característica estructural de las economías. En la mayor parte de los casos parte de los acreedores pertenecen al sector privado, por lo que es necesario establecer un mecanismo internacional de reestructuración de la deuda soberana.

- Otras medidas para ampliar el espacio fiscal y mejorar las condiciones de acceso al financiamiento son la revisión de las leyes de responsabilidad y reglas fiscales procíclicas - que solo se enfocan en el gasto y no en los ingresos- y lograr que las agencias calificadoras consideren en sus metodologías de evaluación de riesgo que el deterioro en las posiciones fiscales de los países para enfrentar la crisis es extraordinario y temporal. 
- Por su parte, los bancos centrales de la región han anunciado "medidas pocos convencionales" para expandir la liquidez, incluida la compra de activos públicos y privados. Esto hace necesaria una mayor coordinación entre las autoridades fiscales y monetarias para financiar los paquetes de estímulos anunciados, sea mediante la compra de títulos emitidos por el sector público directamente, sea mediante el uso de activos externos para financiar el incremento del gasto.

- Se requiere coordinación entre los actores para determinar la manera y el tiempo para "normalizar" la política monetaria en la región. También es necesario aumentar la coordinación entre la política fiscal y la política monetaria, así como entre los bancos centrales y los entes de supervisión y regulación bancaria para garantizar que estos recursos sean canalizados a los hogares y a los sectores productivos que lo necesiten y, que no pasen a formar parte de las reservas de las instituciones financieras o a alimentar la demanda de activos externos.

- Dada la magnitud de los choques es necesario adecuar las herramientas macroprudenciales empleadas para atenuar la volatilidad cambiaria, y las depreciaciones excesivas de las monedas. El acceso a divisas, particularmente a dólares, se ha vuelto cada vez más limitado para los países en desarrollo y los mercados emergentes. Aunque el establecimiento de líneas de intercambio (swaps) entre la Reserva Federal de los Estados Unidos y los bancos centrales del Brasil y México es un primer paso, el resto de la región se encuentra fuera de ese mecanismo de apoyo.

- Una forma adicional de ampliar el acceso a la liquidez en dólares estadounidenses de todos los países sería mediante una emisión a gran escala de derechos especiales de giro (DEG), posiblemente mayor a la efectuada en 2009, cuando el FMI emitió DEG por 250.000 millones de dólares para ayudar a combatir la crisis financiera mundial, conforme a la propuesta del G20.

\section{F. Efectos estructurales de la pandemia: el mundo posterior} al COVID-19

- La pandemia ha alterado las relaciones económicas y sociales de un modo radical y sus consecuencias trascenderán su duración. Es altamente probable que sea un catalizador de los cambios que se observaban en la configuración de la producción y el comercio a nivel mundial en la última década.

- El modelo de globalización basado en redes internacionales de producción con una elevada dispersión geográfica mostraba signos de agotamiento visibles en el estancamiento secular de muchas economías, la pérdida de dinamismo del comercio y la alta volatilidad desde la crisis financiera mundial.

- Más recientemente, las restricciones sin precedentes al transporte internacional adoptadas por numerosos países han ocasionado grandes perturbaciones en las cadenas globales de valor.

- Desde la irrupción del COVID-19, las nuevas órdenes manufactureras mundiales han tenido la caída más rápida de los últimos 11 años. El índice global del sector manufacturero (índice de gestores de compras (PMI)) del primer trimestre de 2020 confirma un deterioro de la producción mundial: solo en febrero, la producción manufacturera mundial se derrumbó 7 puntos (IHS Markit, 2020).

- Si bien China ha retomado gradualmente las actividades productivas, enfrenta limitaciones desde el punto de vista de la oferta por la reducción de las importaciones desde Europa y los Estados Unidos, y una demanda mundial muy debilitada, lo que no hace prever una pronta recuperación de la manufactura mundial. Las existencias mundiales de insumos cayeron bruscamente como resultado de las limitaciones de la oferta y muchos países están informando de falta de insumos, especialmente en los sectores que producen bienes para hacer frente a la emergencia sanitaria.

- En suma, la expansión de la crisis productiva ha puesto de manifiesto las vulnerabilidades de la interdependencia, a nivel de países y empresas, y puede llevar a cambios significativos en la organización de la producción a nivel mundial.

- En primer lugar, ha quedado en evidencia la vulnerabilidad de las redes internacionales de producción ante fenómenos imprevistos de gran magnitud, y la consecuente necesidad de dotarlas de una mayor resiliencia. Desde la óptica de las empresas multinacionales que lideran esas redes, esto implica diversificar su red de proveedores en términos de países y empresas, 
privilegiar ubicaciones más cercanas a los mercados finales de consumo (nearshoring) y relocalizar procesos críticos cuando sea económicamente factible, mediante el uso de tecnologías como la manufactura aditiva. Hay señales de movimientos en este sentido en las principales economías mundiales:

- El Japón ha destinado 2.200 millones de dólares de su paquete de estímulo económico ante el COVID-19 para ayudar a sus empresas a relocalizar la producción fuera de China.

- En los Estados Unidos, el índice de relocalización alcanzó su valor máximo en 2019 debido a las tensiones comerciales con China, mientras que el peso de México en las importaciones manufactureras aumentó marcadamente respecto de los proveedores asiáticos (Kearney, 2020). Las perturbaciones ocasionadas por el COVID-19 reforzarían a ambas tendencias.

- En la Unión Europea, más de 200 empresas relocalizaron su producción entre 2016 y 2019. El 50\% de los casos de relocalización proviene de actividades deslocalizadas dentro de la propia Unión Europea, mientras que China representa el 32\% y la India el 5\% (Fundación Europea para la Mejora de las Condiciones de Vida y de Trabajo, 2019).

- En segundo lugar, la crisis ha obligado a las empresas a adecuar su funcionamiento interno a las medidas de distanciamiento social. A medida que la robótica, la automatización y el uso masivo de plataformas de teletrabajo se vuelven más eficientes, más baratas y fáciles de implementar, es probable que la automatización de algunos sectores y procesos productivos se acelere, con impactos sobre el empleo.

- En tercer lugar, en el plano de la gobernanza del comercio mundial, el COVID-19 ha profundizado el debilitamiento de la cooperación internacional y del multilateralismo que se observa hace ya algunos años. Así lo muestran las restricciones a la exportación de productos médicos y sanitarios y de alimentos adoptadas por al menos 60 países de todas las regiones. En este contexto, y consistente con un escenario de acortamiento de las redes internacionales de suministro, resulta probable que los esfuerzos de los principales actores del comercio mundial se vuelquen hacia los acuerdos regionales en detrimento de los multilaterales. Tal escenario llevaría a un comercio mundial más fragmentado y con mayor ocurrencia de conflictos, justo cuando la capacidad de la $\mathrm{OMC}$ de resolver las controversias entre sus miembros se encuentra reducida al mínimo.

- En suma, cabe esperar que la pandemia refuerce tendencias que ya se observaban y que apuntan a un menor nivel de interdependencia productiva y comercial entre las principales economías mundiales, en particular entre los Estados Unidos y Europa, por una parte, y China, por la otra. Ese proceso se vería favorecido por los avances en materia de digitalización y robotización, que reducen la importancia relativa de los bajos costos laborales como factor de competitividad. El resultado neto no sería una reversión de la globalización, sino una economía mundial más regionalizada, organizada en torno a tres polos productivos ya existentes: América del Norte, Europa y Asia oriental y sudoriental.

- Para América Latina y el Caribe, la magnitud del impacto y la capacidad de reacción dependerán en gran medida de la estructura productiva de cada economía, de la participación de sus empresas en las cadenas de valor y de las capacidades productivas existentes.

- Como nunca en los últimos 30 años, hoy está abierto a discusión el modelo dominante de inserción de la región en la economía internacional, basado en la especialización en materias primas, manufacturas de ensamblaje y turismo de sol y playa. En efecto, la disrupción de diversas cadenas globales de valor ha mostrado los riesgos que supone la elevada dependencia regional de las manufacturas importadas. Esto ha quedado particularmente de manifiesto con las severas limitaciones a la disponibilidad de productos esenciales para el combate al COVID-19 a raíz de las restricciones impuestas por la mayoría de sus principales proveedores.

- En el sector automotor, la interrupción de las cadenas globales ha generado un retraso en el abastecimiento de insumos, debido al cierre de las plantas en China, Europa y los Estados Unidos. Las 12 empresas automotrices que operan en México anunciaron a finales de marzo que dejarían de trabajar por la carencia de componentes e insumos esenciales, cierre que ha resultado en una caída del $25,5 \%$ del total de las ventas del sector respecto de marzo de 2019 (AMIA, 2020). En el Brasil, la carencia de componentes e insumos también ha resultado en una ralentización de la producción del sector, que en marzo registró una caída del $21,1 \%$ respecto del mismo mes de 2019 (ANFAVEA, 2020). 
- En este contexto, adquiere una relevancia renovada la adopción de políticas industriales que permitan a la región fortalecer sus capacidades productivas y generar nuevas capacidades en sectores estratégicos. Estos esfuerzos podrían verse facilitados en algunos países por la llegada de nuevas inversiones en el marco del acortamiento de las cadenas de suministro de empresas multinacionales, especialmente estadounidenses.

- El contexto internacional posterior al COVID-19 que es posible ir perfilando apunta a una creciente importancia de los procesos de regionalización de la producción. En ese contexto, la integración regional está llamada a desempeñar un papel clave en las estrategias de desarrollo de los países de América Latina y el Caribe. Un mercado integrado de $\mathbf{6 5 0}$ millones de habitantes constituiría un importante seguro frente a perturbaciones de oferta 0 de demanda generadas fuera de la región. Asimismo, permitiría alcanzar la escala requerida para viabilizar nuevas industrias, así como promover redes de producción e investigación compartida entre los distintos países y subregiones.

- Las conclusiones de los análisis precendentes son duras, pero claras:

- La crisis que sufre la región en 2020, con una caída del PIB del 5,3\%, será la peor en toda su historia (véase el gráfico 12). Para encontrar una contracción de magnitud comparable hace falta retroceder hasta la Gran Depresión de 1930 (-5\%) o más aún hasta 1914 (-4,9\%).

- Los efectos de mediano plazo en materia de reorganización de la producción y del comercio internacional en términos de localización y tecnológicos son importantes. Los escenarios posibles que aún están abiertos son por lo menos tres: continuación de la globalización, pero sobre la base de nuevos modelos de gobernanza más receptivos al multilateralismo y la corrección de las desigualdades entre países; soluciones de alcance exclusivamente nacional, o una acentuación de la regionalización.

- Para la gran mayoría de los países de América Latina y el Caribe, las soluciones de alcance exclusivamente nacional no serían viables por razones de economías de escala, tecnológicas y de aprendizaje.

- Es posible que la mejor solución sea una nueva globalización con una gobernanza proclive a la inclusión y la sostenibilidad, pero para participar activamente en esa nueva globalización, América Latina y el Caribe debe integrarse productiva, comercial y socialmente. Para ello, la coordinación de nuestros países en materia macroeconómica y productiva es crucial para negociar las condiciones de la nueva normalidad, particularmente en una dimensión urgente en la actual crisis y en el mediano plazo: la del financiamiento para un nuevo estilo de desarrollo con igualdad y sostenibilidad ambiental.

Gráfico 12 | América Latina: tasa de crecimiento anual del PIB en términos reales, 1901-2020 (En porcentajes)

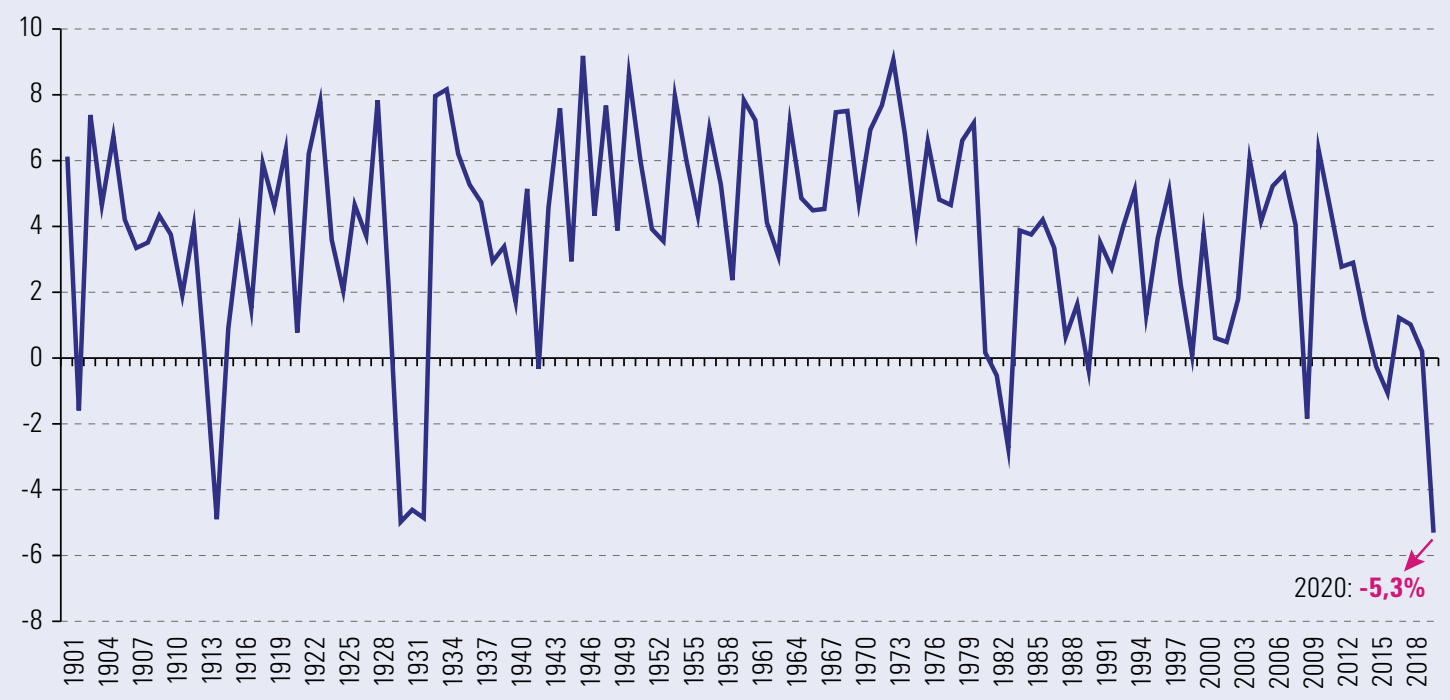

Fuente: Comisión Económica para América Latina y el Caribe (CEPAL) sobre la base de datos de Groningen Growth and Development Centre, Maddison Project Database [en línea] https://www.rug.nl/ggdc/historicaldevelopment/maddison/releases/maddison-project-database-2018 hasta 1950 y cifras oficiales desde 1950 hasta 2019.

Nota: Respecto de 2020 se considera la proyección de la CEPAL. 


\section{Bibliografía}

AMIA (Asociación Mexicana de la Industria Automotriz) (2020), “Reporte de ventas de vehículos ligeros nuevos por marca en marzo de 2020", 2 de abril [en línea] www.amia.com.mx.

ANFAVEA (Asociación Nacional de Fabricantes de Vehículos Automotores) (2020), "Avanço da Covid-19 derruba números da indústria automotiva em quase $90 \%$ no final de março", 6 de abril [en línea] http:// www.anfavea.com.br/docs/Release \% 20-\%20Avan\%C3\%A70\%20da\%20Covid-19\%20derruba\%20 n\%C3\%BAmeros \%20da\%20ind\%C3\%BAstria\%20automotiva\%20em\%20quase \% 2090\%20no\%20 final\%20de\%20mar\%C3\%A7o.pdf.

BPI (Banco de Pagos Internacionales) (2020), "Debt securities statistics" [en línea] https://www.bis.org/ statistics/secstats.htm.

CEPAL (Comisión Económica para América Latina y el Caribe) (2020), "América Latina y el Caribe ante la pandemia del COVID-19: efectos económicos y sociales", Informe Especial COVID-19, № 1, 3 de abril, Santiago.

FMI (Fondo Monetario Internacional) (2019), Regional Economic Outlook. Western Hemisphere: Stunted by Uncertainty, Washington, D.C.

Fundación Europea para la Mejora de las Condiciones de Vida y de Trabajo (2019), "European Reshoring Monitor" [en línea] https://reshoring.eurofound.europa.eu/.

IHS Markit (2020), "Manufacturing downturn deepens outside of China", 1 de abril [en línea] https:// insmarkit.com/research-analysis/manufacturing-downturn-deepens-outside-of-china-April2020.html.

IIF (Instituto de Finanzas Internacionales) (2020a), Capital Flows Report: Sudden Stop in Emerging Markets, 9 de abril.

(2020b), "Global Debt Monitor: COVID-19 lights a fuse", 6 de abril [en línea] https://www.iif.com/ Portals/0/Files/content/Research/Global\%20Debt\%20Monitor_April2020.pdf.

(2020c), "LatAm views: deep recession", 13 de abril [en línea] https://www.iif.com/Portals/0/Files/ content/Research/04_13_2020_latam_views.pdf.

Kearney (2020), "Trade war spurs sharp reversal in 2019 Reshoring Index, foreshadowing COVID-19 test of supply chain resilience" [en línea] https://www.kearney.com/operations-performance-transformation/ us-reshoring-index.

Oficina del Censo de los Estados Unidos (2018), "American Community Survey (ACS)" [en línea] https:// www.census.gov/programs-surveys/acs.

OMT (Organización Mundial del Turismo) (2020), "Impact assessment of the COVID-19 outbreak on international tourism", 24 de marzo [en línea] https://webunwto.s3.eu-west-1.amazonaws.com/s3fspublic/2020-03/24-03Coronavirus.pdf.

Pineda, E. y A. Musacchio (2020), "Solving the state-owned enterprises puzzle in Latin America and the Caribbean", Banco Interamericano de Desarrollo (BID), 18 de enero [en línea] https://blogs.iadb.org/ gestion-fiscal/en/solving-the-state-owned-enterprises-puzzle-in-latin-america-and-the-caribbean.

Este Informe Especial es el segundo de una serie que elaborará la Comisión Económica para América Latina y el Caribe (CEPAL) sobre la evolución y los efectos de la pandemia del COVID-19 en América Latina y el Caribe. Sus análisis económicos y sociales se actualizarán a medida que surja información relevante. La Secretaria Ejecutiva de la CEPAL, Alicia Bárcena, dirige la elaboración de este Informe, con el apoyo técnico de la Oficina del Secretario Ejecutivo Adjunto, Mario Cimoli, las Divisiones sustantivas encargadas de los temas que aquí se tratan, y las sedes subregionales y oficinas nacionales de la CEPAL.

Copyright (C) Naciones Unidas, 2020 